\title{
Temperature and density structure of a recurring active region jet ${ }^{\star}$
}

\author{
Sargam M. Mulay, Giulio Del Zanna, and Helen Mason
}

\begin{abstract}
DAMTP, Centre for Mathematical Sciences, University of Cambridge, Wilberforce Road, Cambridge, CB3 0WA, UK
e-mail: smm96@cam.ac.uk
\end{abstract}

Received 26 April 2016 / Accepted 11 September 2016

\begin{abstract}
Aims. We present a study of a recurring jet observed on October 31, 2011 by the Atmosphereic Imaging Assembly (AIA) on board the Solar Dynamic Observatory, the X-ray Telescope (XRT) and EUV Imaging Spectrometer (EIS) on board Hinode. We discuss the physical parameters of the jet that are obtained using imaging and spectroscopic observations, such as density, differential emission measure, peak temperature, velocity, and filling factor.

Methods. A differential emission measure (DEM) analysis was performed at the region of the jet spire and the footpoint using EIS observations and also by combining AIA and XRT observations. The resulting EIS DEM curves were compared to those obtained with AIA-XRT. The DEM curves were used to create synthetic spectra with the CHIANTI atomic database. The predicted total count rates for each AIA channel were compared with the observed count rates. The effects of varying elemental abundances and the temperature range for the DEM inversion were investigated. Spectroscopic diagnostics were used to obtain an electron number density distribution for the jet spire and the jet footpoint.

Results. The plasma along the line of sight in the jet spire and jet footpoint was found to be peak at $2.0 \mathrm{MK}(\log T[\mathrm{~K}]=6.3)$. We calculated electron densities using the Fe XII $(\lambda 186 / \lambda 195)$ line ratio in the region of the spire $\left(N_{\mathrm{e}}=7.6 \times 10^{10} \mathrm{~cm}^{-3}\right)$ and the footpoint $\left(1.1 \times 10^{11} \mathrm{~cm}^{-3}\right)$. The plane-of-sky velocity of the jet is found to be $524 \mathrm{~km} \mathrm{~s}^{-1}$. The resulting EIS DEM values are in good agreement with those obtained from AIA-XRT. The synthetic spectra contributing to each AIA channel confirms the multi-thermal nature of the AIA channels in both regions. There is no indication of high temperatures, such as emission from Fe XVII $(\lambda 254.87)(\log T[\mathrm{~K}]=6.75)$ seen in the jet spire. In the case of the jet footpoint, synthetic spectra predict weak contributions from Ca XVII ( $\lambda 192.85)$ and Fe XVII ( 2254.87$)$. With further investigation, we confirmed emission from the Fe XVIII (93.932 $⿱$ ) line in the AIA $94 \AA$ channel in the region of the footpoint. We also found good agreement between the estimated and predicted Fe XVIII count rates. A study of the temporal evolution of the jet footpoint and the presence of high-temperature emission from the Fe XVIII $(\lambda 93.932)(\log T[\mathrm{~K}]=6.85)$ line leads us to conclude that the hot component in the jet footpoint was present initially and that the jet had cooled down by the time EIS observed it.
\end{abstract}

Key words. Sun: corona - Sun: atmosphere - Sun: transition region - Sun: UV radiation

\section{Introduction}

Solar jets are small-scale ubiquitous transients observed as collimated flows of plasma in and at the boundary of coronal holes (coronal hole $(\mathrm{CH})$ jets) and also at the edge of active regions (active region (AR) jets). Active region jets have been observed in $\mathrm{H} \alpha$, extreme-ultraviolet (EUV; see Innes et al. 2011; Zhang \& Ji 2014a,b; Mulay et al. 2016) and X-ray wavelengths (cf. Chifor et al. 2008b,a) using ground-based and space-based instruments.

It has been observed that AR jets are often associated with nonthermal type III radio bursts (Kundu et al. 1995; Innes et al. 2011; Chandra et al. 2015; Mulay et al. 2016). The energetic particles follow the field lines that are open to the heliosphere and can produce impulsive, electron $/{ }^{3} \mathrm{He}$-rich, solar energetic particle (SEP) events in the interplanetary medium (Nitta et al. 2015; Innes et al. 2016). Therefore, AR jets and their associated phenomena are one of the important features involved in spaceweather studies.

Using imaging and spectroscopic observations at a number of wavelengths, we can probe different layers of the solar atmosphere and study the temperature structure of jets in

\footnotetext{
* Two movies attached to Fig. 2 are available at http://www. aanda.org
}

detail. Until recently, it was difficult to carry out a detailed study of jets because of the limited spatial and temporal resolution of early instruments. The high spatial and temporal resolution of the Atmospheric Imaging Assembly (AIA; Lemen et al. 2012) on the Solar Dynamic Observatory (SDO) and the X-ray Telescope (XRT; Golub et al. 2007) imaging instrument on Hinode, together with the spectral capabilities of EUV Imaging Spectrometer (EIS; Culhane et al. 2007) on Hinode have enabled us to carry out an in-depth analysis.

A number of authors have studied the physical parameters such as velocities, density, size, location and direction of AR jets using EUV imaging observations (Innes et al. 2011; Kayshap et al. 2013; Chen et al. 2013; Mulay et al. 2016). There are also a few results available from spectroscopic observations (Kim et al. 2007; Chifor et al. 2008b; Yang et al. 2011; Madjarska 2011; Matsui et al. 2012; Lee et al. 2013). Kim et al. (2007); Chifor et al. (2008b); and Yang et al. (2011) studied AR jets using simultaneous EIS and XRT observations. They found that EUV and SXR jets had similar projected speeds, lifetimes, and sizes and they also observed that EUV jets had the same location, direction, and collimated shape as the SXR jets.

Using spectroscopic observations from EIS, Kim et al. (2007) observed a jet footpoint; Matsui et al. (2012) and Lee et al. (2013) observed a jet for the temperature range from 
$\log T[\mathrm{~K}]=4.9$ to 6.3 in their individual study of AR jets. Yang et al. (2011) reported the jet plasma temperature ranges from $\log T[\mathrm{~K}]=4.7$ to 6.3 , i.e. from 0.05 to $2.0 \mathrm{MK}$, and maximum electron densities ranging from $N_{\mathrm{e}}=6.6 \times 10^{9}$ to $N_{\mathrm{e}}=3.4 \times 10^{10} \mathrm{~cm}^{-3}$; whereas Chifor et al. (2008b) reported a jet temperature ranges from $\log T[\mathrm{~K}]=5.4$ to $\log T[\mathrm{~K}]=6.4$ and found density above $\log N_{\mathrm{e}}=11 \mathrm{~cm}^{-3}$.

The temperature distribution of AR jets has been studied using differential emission measure (DEM) methods assuming multi-thermal plasma along the line of sight using EUV imaging observations. Kayshap et al. (2013) studied an AR surge using the DEM method of Aschwanden et al. (2013) and reported an average temperature of $2 \mathrm{MK}$ and a density of $4.1 \times 10^{9} \mathrm{~cm}^{-3}$; whereas using the same DEM method, Chen et al. 2013 studied another AR jet and reported a high temperature $(7 \mathrm{MK})$ in the footpoint region. A recent study of $20 \mathrm{AR}$ jets by Mulay et al. (2016) using multiwavelength AIA observations reported the temperature of the jet spire ranging from $\log T$ $[\mathrm{K}]=6.2$ to 6.3 and the electron density ranging from $8.6 \times 10^{9}$ to $1.3 \times 10^{10} \mathrm{~cm}^{-3}$. They also investigated the temperature structure at the region of the footpoint, which was found to peak at log $T[\mathrm{~K}]=6.5$ with electron number density ranging from $8.4 \times 10^{9}$ to $1.1 \times 10^{10} \mathrm{~cm}^{-3}$.

All the above studies provided various physical parameters of AR jets, but a detailed investigation of the temperature structure in the region of the spire and the footpoint of AR jets using simultaneous imaging and spectroscopic observations has remained elusive.

Even with existing instruments, it has been a challenge to find simultaneous imaging and spectroscopic observations of an active region jet. After a careful search through available data sets, we have found suitable observations of a recurrent jet originating from the periphery of an active region. To the best of our knowledge, this is the first comprehensive investigation of the temperature structure of the jet spire and the jet footpoint of an AR recurrent jet using simultaneous imaging and spectroscopic observations. In this study, we focus on two instances of a recurrent jet where we individually observed the spire and footpoint of a jet.

In Sect. 2, we present our observations and describe the instruments from which the data has been taken for this study. Section 3 describes the DEM analysis techniques used to investigate the temperature structure of the jet spire and the footpoint. We also discuss the temporal evolution of the footpoint region and the change in temperature during that period. In Sect. 4, we discuss and summarise our results.

\section{Observations}

In this section, we discuss observations of a recurrent active region jet made using SDO/AIA, Hinode/XRT and Hinode/EIS instruments. We describe below the instrument specifications and data processing techniques.

\subsection{Atmospheric Imaging Assembly (AIA)}

We studied a recurrent jet observed on October 31, 2011 originating from the western edge of an active region NOAA 11330 (N08 W49). The AIA observed a series of recurrent jets from 13:00 to 18:00 UT (see online Movie 1) in all EUV/UV channels with its high spatial (about $\sim 1.2^{\prime \prime}$ resolution, $0.6^{\prime \prime}$ per pixel) and temporal (12 s) resolution. We used six EUV wavelength channels (in brackets we indicate the spectral lines dominating each channel): $94 \AA$ (Fe X, Fe XIV, Fe XVIII), $131 \AA$ (Fe VIII, Fe XXI), $171 \AA$ (Fe IX), $193 \AA$ (Fe XII, Ca XVII, Fe XXIV), $211 \AA$ (Fe XIV), and $335 \AA$ (Fe XVI), which are sensitive to a range of coronal temperatures (see O'Dwyer et al. 2010; Del Zanna et al. 2011; 2013b, for a detailed description of the EUV filters in the AIA and Del Zanna et al. 2015, for their temperature responses). Full disk AIA level 1.0 data were downloaded and processed to level 1.5 using the standard AIA software package (aia_prep.pro) available in the SolarSoft (SSW; Freeland \& Handy 1998) libraries. Further, the data were normalised by the exposure time. We carefully visually co-aligned images from different EUV channels by comparing the solar features, such as a moss region and bright patches.

\subsection{X-ray Telescope (XRT)}

These jets were also observed in X-ray wavelength channels Ti-poly (see online Movie 2) and Be-thin of the XRT instrument on board the Hinode satellite (Kosugi et al. 2007). We obtained high resolution (1.02") images from Ti-poly and Be-thin filters with a temporal resolution of $1 \mathrm{~min}$ and $\sim 15 \mathrm{~min}$, respectively. The XRT data were downloaded and processed using the standard XRT software package (xrt_prep.pro; Kobelski et al. 2014) available in the SSW libraries. The processing includes the subtraction of a model dark frame, correction for vignetting, and removal of high-frequency pattern noise. Further, we normalised the X-ray images by their exposure times. We employed an updated XRT filter calibration (Narukage et al. 2011) in this analysis.

\subsection{EUV imaging spectrometer (EIS)}

The EUV Imaging Spectrometer (EIS; Culhane et al. 2007) on board Hinode provides high resolution spectra in two wavelength bands, 170-211 $\AA$ (SW: short wavelength) and 246$292 \AA$ (LW: long wavelength). The EIS study YK_AR_50×384 was run from 14:00 UT to 17:42 UT on October 31, 2011. The raster covered a $50 \times 384 \operatorname{arcsec}^{2}$ area on the Sun in $18 \mathrm{~min}$. For each raster, the $1^{\prime \prime}$ slit scanned 51 positions from west-to-east (in the opposite direction of solar rotation) with exposure times of $20 \mathrm{~s}$. This study includes eight spectral windows. The EIS raster observed the jet spire during its fourth raster scan (14:56:0215:14:05 UT) and the jet footpoint during its ninth raster scan (16:28:17-16:46:20 UT). Tables 1 and 2 provide details of the EIS observation and observed spectral lines.

Figure 1 shows the AIA $193 \AA$ image of the active region NOAA 11330 (shown with a white arrow) observed on October 31, 2011 at 14:56 UT. The white box shows the EIS raster field of view and the red box indicates the field of view shown in Fig. 2. A moss region, shown with a yellow box, was used to verify our method of analysis (see Appendix).

The EIS data were processed using the standard processing routine (eis_prep.pro) provided in the SSW to obtain calibrated intensities in units of erg cm $\mathrm{cm}^{-2} \mathrm{~s}^{-1} \mathrm{sr}^{-1} \AA^{-1}$ at each pixel in the data set. This routine flags saturated data and removes dark current, hot pixels, and cosmic ray hits. The cfit routine was applied to all of the lines at each pixel in the EIS raster. The offset (18 pixels) between the SW and LW CCD channels in the solar $Y$ direction was corrected. We employed the radiometric calibration by Del Zanna (2013a) to correct for the degradation of EIS instrument over time. 


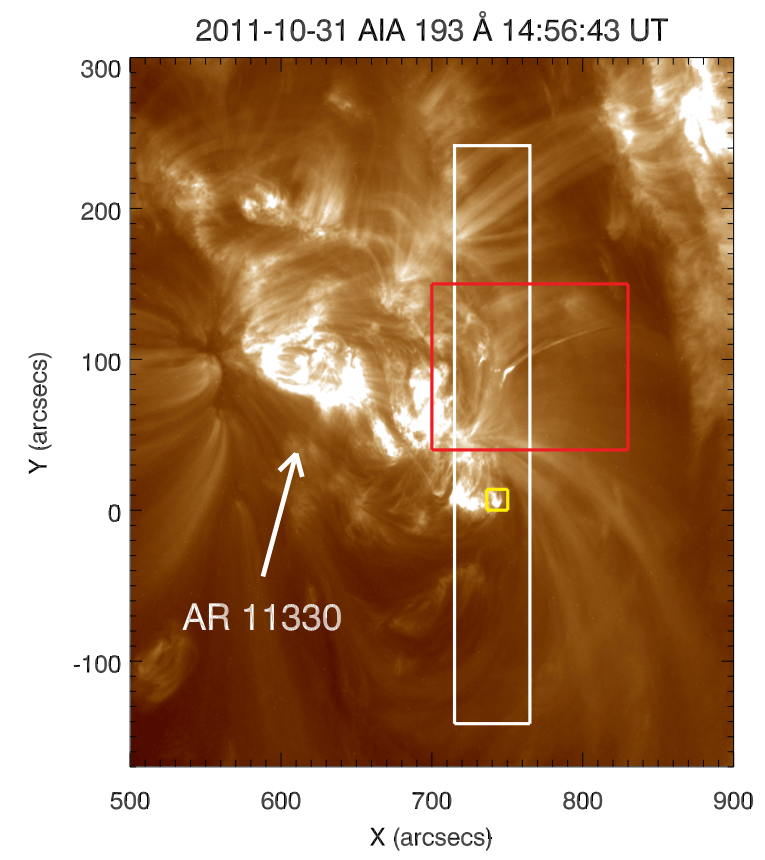

Fig. 1. The Atmospheric Imaging Assembly (AIA) $193 \AA$ image of an active region NOAA 11330 (N08 W49) (shown with a white arrow) observed on October 31, 2011. The white box shows EIS raster field of view and the red box shows the field of view shown in Fig. 2. The yellow box shows a moss region.

\section{Data analysis and results}

\subsection{Overview and kinematics}

The jet showed its recurrent nature at the same location with very similar morphological features from 13:00 UT to 18:00 UT. We also observed plasma-blobs travelling along the jet spire in the AIA channels. Figure 2 shows the recurrent jet (reverse colour image) at 14:56 UT (top panel) and 16:30 UT (bottom panel) in the AIA $171 \AA$ (left panel), XRT-Be-thin (middle panel), and XRT-Ti-poly (right panel) channels. The Eiffel Tower shaped jet in the AIA $171 \AA$ image at 14:56 UT (shown with a black arrow in the top left panel) represents an inverted Y topology of the magnetic field lines associated with the jet footpoint structure. A multi-threaded spire is observed during its evolution, which is clearly seen in the AIA $171 \AA$ image at 16:30 UT (shown with a black arrow in the bottom left panel). The XRT images show a similar morphology for the jet along with a bright spot at the footpoint. The overplotted white boxes on AIA $171 \AA$ images in Fig. 2 show the EIS field of view used in Figs. 3, 6, and 7.

The jet spire and the footpoint were observed over the range of temperatures from $\log T[\mathrm{~K}]=4.7$ to $\log T[\mathrm{~K}]=6.4$ in the EIS observations. The emission from these regions is shown with black arrows in Fig. 3 (second column). Figure 3 shows the EIS raster images (reverse colour) in calibrated units (phot $\mathrm{cm}^{-2} \mathrm{~s}^{-1} \operatorname{arcsec}-2$ ) for the jet spire (top panel) and the jet footpoint (bottom panel) in Mg VI ( $\lambda 268.991$, $\log T[\mathrm{~K}]=5.7)$, Fe VIII $(\lambda 186.605, \log T[\mathrm{~K}]=5.8)$, Fe XII $(\lambda 186.88$ and $\lambda 195.119), \log T[\mathrm{~K}]=6.2)$, and Fe XV $(\lambda 284.160$, $\log T[\mathrm{~K}]=6.4)$ lines, respectively. There is no emission observed in Fe XVII line $(\lambda 254.87)(\log T[\mathrm{~K}]=6.75)$ at the region of the spire and the footpoint.

The highly dynamic activity of the jet was clearly observed in the AIA channels during its evolution (see online Movie 1). Figure 4 (top panel) shows the jet in the AIA $171 \AA$ channel at
Table 1. EIS observation details.

\begin{tabular}{ccc}
\hline \hline EIS details & Jet-spire & Jet-footpoint \\
\hline Raster start time (UT) & $14: 56: 02$ & $16: 28: 17$ \\
Raster end time (UT) & $15: 14: 05$ & $16: 46: 20$ \\
Slit width & $1^{\prime \prime}$ & $1^{\prime \prime}$ \\
Field of view & $50^{\prime \prime} \times 384^{\prime \prime}$ & $50^{\prime \prime} \times 384^{\prime \prime}$ \\
Raster cadence (min) & 18 & 18 \\
Exposure time (s) & 20 & 20 \\
Exposure number & 9,10 & 20,21 \\
Event time (UT) & $14: 58: 55$ & $16: 35: 08$ \\
& and $14: 59: 17$ & and $16: 35: 30$ \\
\hline
\end{tabular}

Table 2. List of the EIS observed emission lines.

\begin{tabular}{ccc}
\hline \hline EIS observed lines & $\begin{array}{c}\lambda \\
{[\AA]}\end{array}$ & $\begin{array}{c}\log T_{\max } \\
{[\mathrm{K}]}\end{array}$ \\
\hline Fe VIII (bl) & 186.605 & 5.8 \\
Fe XII (sbl) & 186.88 & 6.2 \\
Fe XII (sbl) & 195.119 & 6.2 \\
Fe XVII & 254.87 & 6.75 \\
He II + Si X & 256.32 & 4.7 \\
Si X & 256.4 & 6.2 \\
S XIII & 256.686 & 6.5 \\
Si X (bl) & 261.056 & 6.2 \\
Mg VI & 268.991 & 5.7 \\
Al IX (bl) & 284.042 & 6.1 \\
Fe XV (bl) & 284.160 & 6.4 \\
\hline
\end{tabular}

Notes. bl - blended lines; sbl - self-blend lines.

14:58 UT and overplotted yellow box shows the region where the EIS observed an emission from the spire. We obtained a temporal evolution (bottom panel) of this region in all AIA channels to see the variability of the jet-spire structure during its evolution. We also investigated the AIA count rates in the region of the footpoint. Figure 5 (top panel) shows the jet in the AIA $171 \AA$ channel at 15:35 UT and the overplotted green box shows the region where EIS observed the emission from the footpoint. We also obtained the temporal evolution (bottom panel) of this region in all AIA channels and observed the variability in the jet-footpoint region. The overplotted solid lines indicate the EIS slit position start time for two exposures where EIS observed the spire and footpoint and dashed lines indicate the slit position end times. The light curves show a sudden increase in the count rates in all AIA channels during the period for the jet-spire study with EIS (see Fig. 4). For the footpoint study (see Fig. 5), the AIA count rates at the time of EIS observations are past their peak and decreasing steadily.

\subsection{AIA-EIS-XRT co-alignment}

A direct comparison of AIA images with EIS spectroscopic data is not straightforward for several reasons: the AIA channels are multi-thermal, the AIA and EIS pixel size and spatial resolution differ, the EIS slit scans the field of view to produce a raster, and the exposure times for the AIA images and EIS raster scan also differ.

The AIA $193 \AA$ channel, which is dominated by Fe XII, is most suitable for a direct comparison. EIS observes the Fe XII line $(\lambda 195.119, \log T[\mathrm{~K}]=6.2)$ in the SW channel. We followed a procedure given by Del Zanna et al. (2011) to 

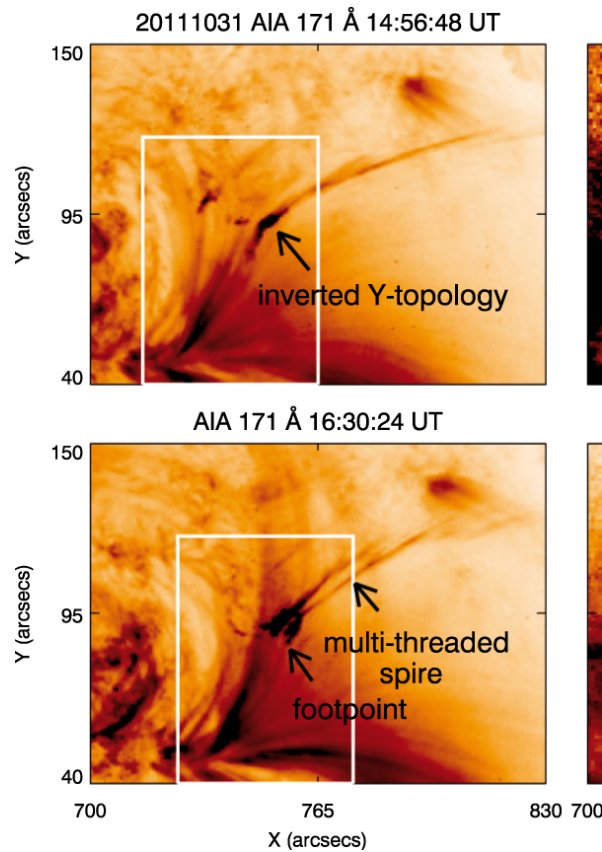

XRT Be Thin 14:56:47 UT

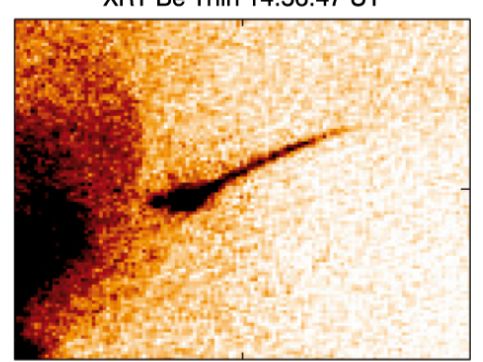

XRT Be Thin 16:30:20 UT

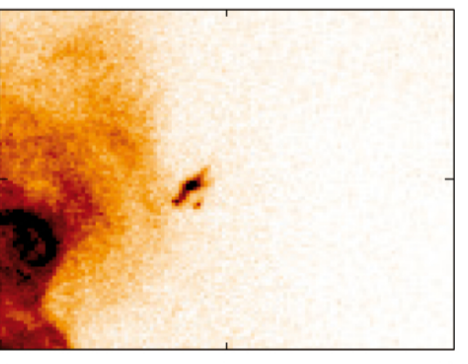

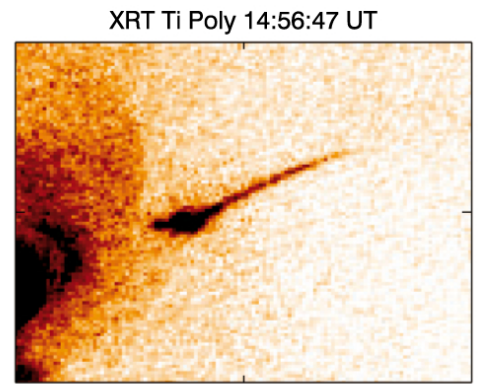

XRT Ti Poly 16:30:32 UT

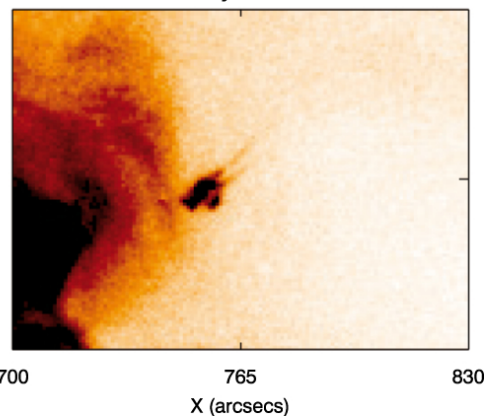

Fig. 2. Recurrent jet observed on October 31, 2011 originating from the western edge of an active region NOAA 11330 (N08 W49). The jet at 14:56 UT (top panel) and at 16:30 UT (bottom panel) in the AIA $171 \AA$ A channel (left panel) of the AIA instrument and in the Be-thin (middle panel) and Ti-poly filters (right panel) of the XRT instrument (reverse colour images). The overplotted white boxes on the AIA $171 \AA$ images show the EIS field of view used in Figs. 3, 6, and 7 (see Movie 1 and Movie 2).

co-align the images from the AIA $193 \AA$ channel with the EIS Fe XII raster: firstly, we convolve each simultaneous AIA $193 \AA$ image with each EIS slit position by considering a Gaussian PSF of $2^{\prime \prime}$ full width half maximum (FWHM). The co-temporal AIA images taken during the EIS exposures are averaged and then rebinned to the EIS pixel size (in this case, it is $1^{\prime \prime}$ ) to obtain a slice of corresponding averaged AIA image. We then built a time-averaged rebinned map of AIA $193 \AA$ channel. The data from the other AIA channels together with the XRT channels are co-aligned according to emission observed in the AIA $193 \AA$ channel. In this analysis, we used only the high cadence (1 min) images from the XRT Ti-poly filter. We also found an offset of 12 pixels in the $X$ direction and 36 pixels in the $Y$ direction between the rebinned AIA maps and EIS raster maps, and we corrected all the maps.

Figure 6 shows the jet spire and the jet footpoint observed with EIS Fe VIII and Fe XII, time-averaged rebinned maps in the AIA 131 and $193 \AA$ channels, and XRT Ti-poly filter. The two white overplotted boxes (shown with black arrows in Col. 1) indicate the region of the spire and the footpoint used for the DEM analysis (see Sect. 3.6). The jet spire appears as a small blob in the Fe VIII and Fe XII raster images. The time-averaged AIA $193 \AA$ images are in very good agreement with the emission seen in Fe XII by EIS. Also, the AIA $131 \AA$ image shows a similar structure and morphology for the jet as in the EIS Fe VIII observation, indicating that the emission observed in the AIA $131 \AA$ comes primarily from the lower temperature contribution, mainly Fe VIII.

\subsection{Spectroscopic techniques}

We used spectroscopic techniques to derive the basic physical plasma parameters such as electron density, differential emission measure, and filling factor.
The intensity of an optically thin emission line can be written as

$I=0.83 A b(z) \int G\left(T_{\mathrm{e}}, N_{\mathrm{e}}\right) N_{\mathrm{e}}^{2} \mathrm{~d} h$,

where the factor 0.83 is the ratio of protons to free electrons, $A b(z)$ is the elemental abundances, $N_{\mathrm{e}}$ is the electron number density, and $h$ is the column depth of the emitting plasma along the line of sight. The value $G\left(T_{\mathrm{e}}, N_{\mathrm{e}}\right)$ is the contribution function, which contains all the atomic parameters for each spectral line and is defined as

$G\left(T_{\mathrm{e}}, N_{\mathrm{e}}\right)=\frac{h c}{4 \pi \lambda_{i, j}} \frac{A_{j i}}{N_{\mathrm{e}}} \frac{N_{j}\left(X^{+m}\right)}{N\left(X^{+m}\right)} \frac{N\left(X^{+m}\right)}{N(X)}$,

where $i$ and $j$ are the lower and upper levels, $A_{j i}$ is the spontaneous transition probability, $\frac{N_{j}\left(X^{+m}\right)}{N\left(X^{+m}\right)}$ is the population of level $j$ relative to the total $N\left(X^{+m}\right)$ number density of ion $X^{+m}$ and is a function of electron temperature and density, and $\frac{N\left(X^{+m}\right)}{N(X)}$ is the ratio of the ion $X^{+m}$ relative to the total number density of element X.

A differential emission measure $\operatorname{DEM}(\mathrm{T})$ can be defined as

$\int_{T} \operatorname{DEM}(T) \mathrm{d} T=\int N_{\mathrm{e}}^{2} \mathrm{~d} h$,

where DEM represents the amount of plasma along the line of sight that emits observed radiation and has a temperature between $T$ and $T+\mathrm{d} T$. The emission measure (EM) can be calculated by integrating the DEM over a given temperature range. For an individual ion, this temperature range is about $0.3($ in $\log T)$,

$E M=\int N_{\mathrm{e}}^{2} \mathrm{~d} h=\int_{T} \operatorname{DEM}(T) \mathrm{d} T\left[\mathrm{~cm}^{-5}\right]$.

The above equation is valid only if the volume is completely filled by the emitting plasma, otherwise a filling factor should be 

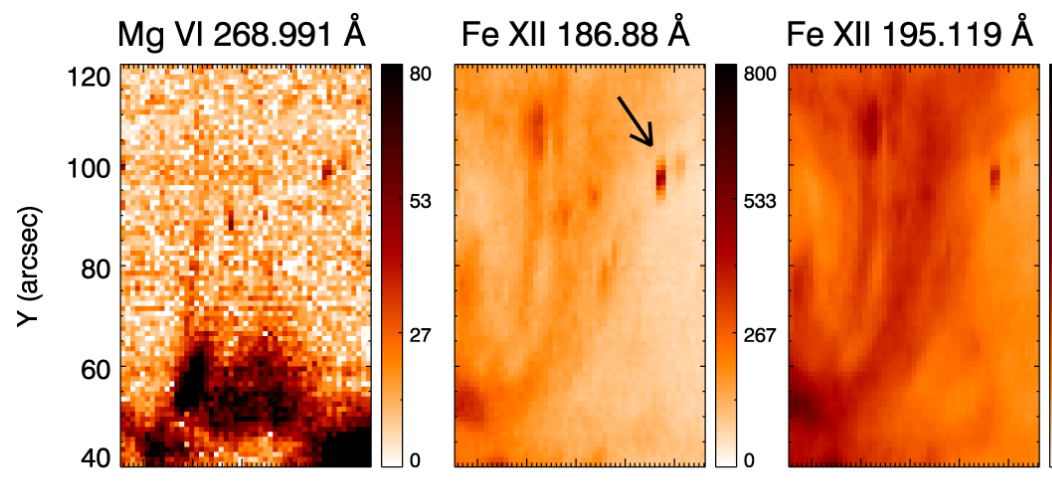

720730740750760
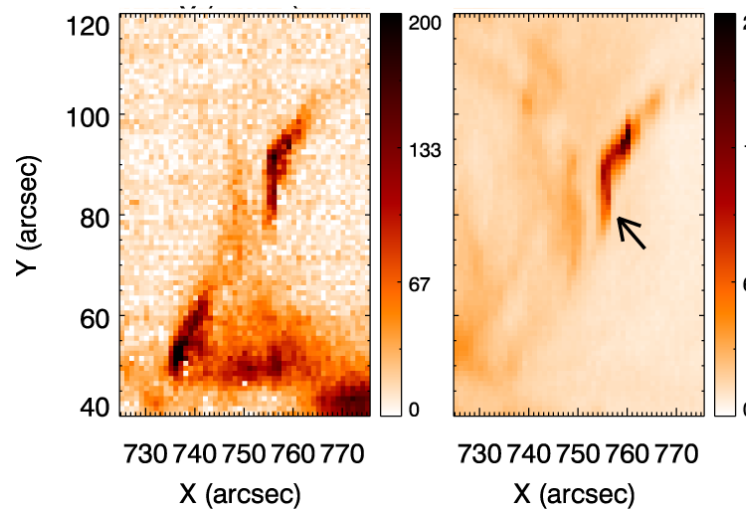

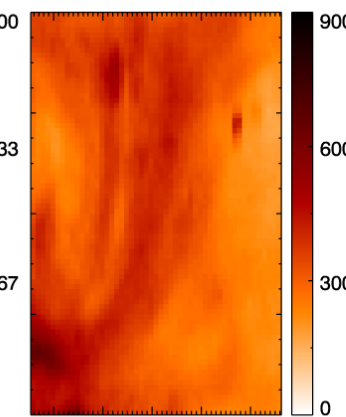

720730740750760

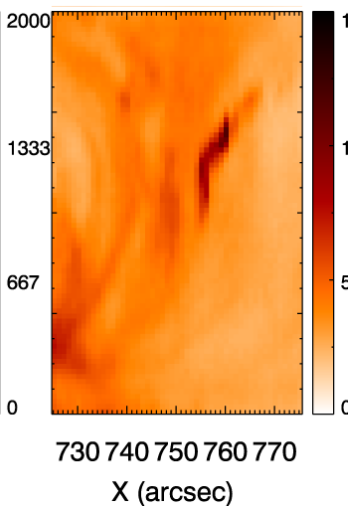

Fe VIII $186.6 \AA$

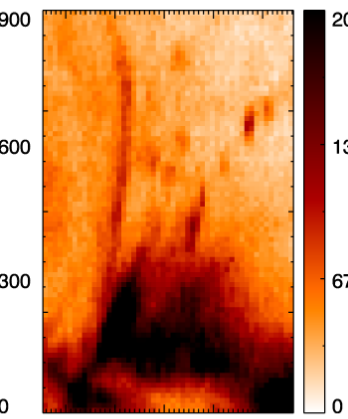

720730740750760

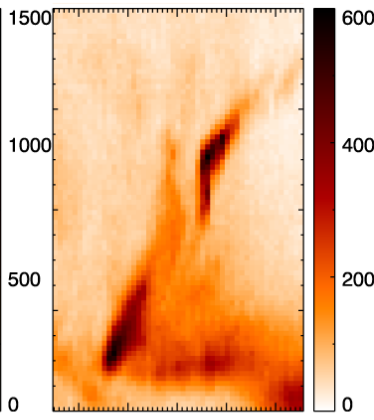

730740750760770

$\mathrm{X}(\operatorname{arcsec})$

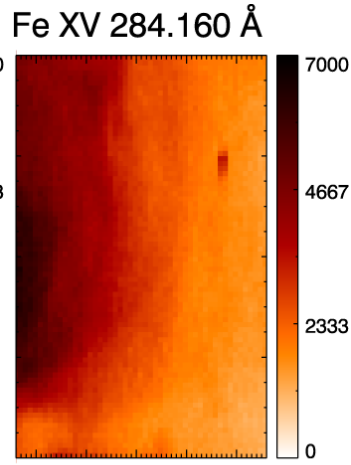

720730740750760

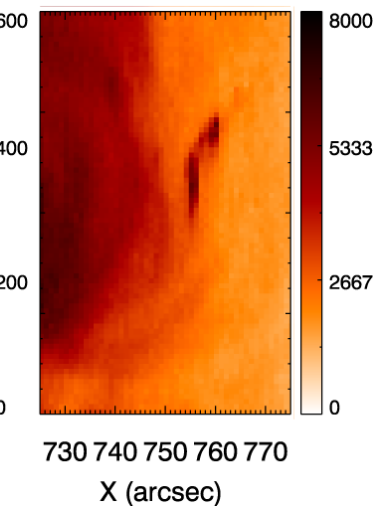

Fig. 3. EIS raster images (reverse colour) showing the morphological features observed during the recurrent jet event in Mg VI, Fe VIII, Fe XII, and Fe XV lines. The jet spire (observed during 14:56:02-15:14:05 UT) (top panel) and the jet footpoint (observed during 16:28:17-16:46:20 UT) (bottom panel) are shown with arrows in the Fe XII images, respectively. The colour bars for the EIS rasters indicate the actual calibrated units $\left(\right.$ phot cm $\left.{ }^{-2} \mathrm{~s}^{-1} \operatorname{arcsec}^{-2}\right)$.

used. The filling factor of a plasma $(\phi)$ is the fraction of a volume that is contributing to the observed emission,

$\phi=\frac{E M}{N_{\mathrm{e}}^{2} h}$,

where $E M$ is measured using Eq. (4) and $\mathrm{N}_{\mathrm{e}}$ is measured by the line ratio techniques (see Sect. 3.4).

\subsection{Density measurement}

The ratio of intensities for certain pairs of emission lines emitted from the same ion, for example some coronal ions, can be used as a density diagnostic. Owing to a broad range of sensitivity of the Fe XII lines $\left(\log N_{\mathrm{e}}=8-12\right)$, we used the density-sensitive pair ratio $(\lambda 186 / \lambda 195)$ to calculate an electron density profile in the jet. We used theoretical line intensity ratios of Fe XII $(\lambda(186.854+186.887) / \lambda(195.119+195.179))$ lines from the CHIANTI atomic database v.8 (Dere et al. 1997; Del Zanna et al. 2015).

Figure 7 (top panel) curve represents the theoretical line intensity ratio as a function of electron density. The density ratio for the jet spire and jet footpoint are shown with red and blue boxes, respectively. The vertical lines overplotted on the curve show the errors estimated by considering $20 \%$ uncertainty (accounting for uncertainties in the atomic data) in the calibrated intensities. The bottom panel shows Fe XII density maps for the jet spire (a) and the jet footpoint (b). The emission was observed for two EIS raster positions shown with orange arrows.

We calculated electron densities for the jet spire and the jet footpoint, (at the locations given by the green boxes in Fig. 7, bottom panel, shown with orange arrows), which are found to be
$N_{\mathrm{e}}=7.6 \times 10^{10}$ and $1.1 \times 10^{11} \mathrm{~cm}^{-3}$, respectively. We note that with $20 \%$ error bars these values could be in the high density limit. We used these density values in the DEM analysis of the spire and the footpoint (see Sect. 3.6).

From the observation we found that the $\lambda 186 / \lambda 195$ pair ratio is greater than 1.2 in parts of the jet, shown with a white arrow in the density map (Fig. 7 bottom panel (b)). This indicates that the region has a high density $\left(\log N_{\mathrm{e}} \geq 11.5\right)$, which is above the range of sensitivity of Fe XII lines ratio diagnostics.

\subsection{Velocity measurement}

We performed a time-distance analysis to obtain the plane-of-sky velocity of the jet using the AIA $171 \AA$ channel images (dominated by Fe IX; $\log T[\mathrm{~K}]=5.8$ ). Figure 8 (top panel) shows the AIA $171 \AA$ image of the jet and the white line represents an artificial slit along the direction of the jet spire that is used to obtain the time-distance plot. The bottom panel shows the jet activity for an hour from 14:30 to 15:30 UT. The near vertical features in the time-distance plot shows recurrent jets originating from the same location with similar morphology. We calculated a velocity for the jet at 14:58:55 UT (the same time when EIS observed the jet-spire) and it was found to be $524 \mathrm{~km} \mathrm{~s}^{-1}$ initially.

\subsection{Differential emission measure (DEM)}

We performed a DEM analysis to determine the distribution of plasma as a function of temperature. In this section, we present our results from the DEM analysis using EIS spectroscopic 

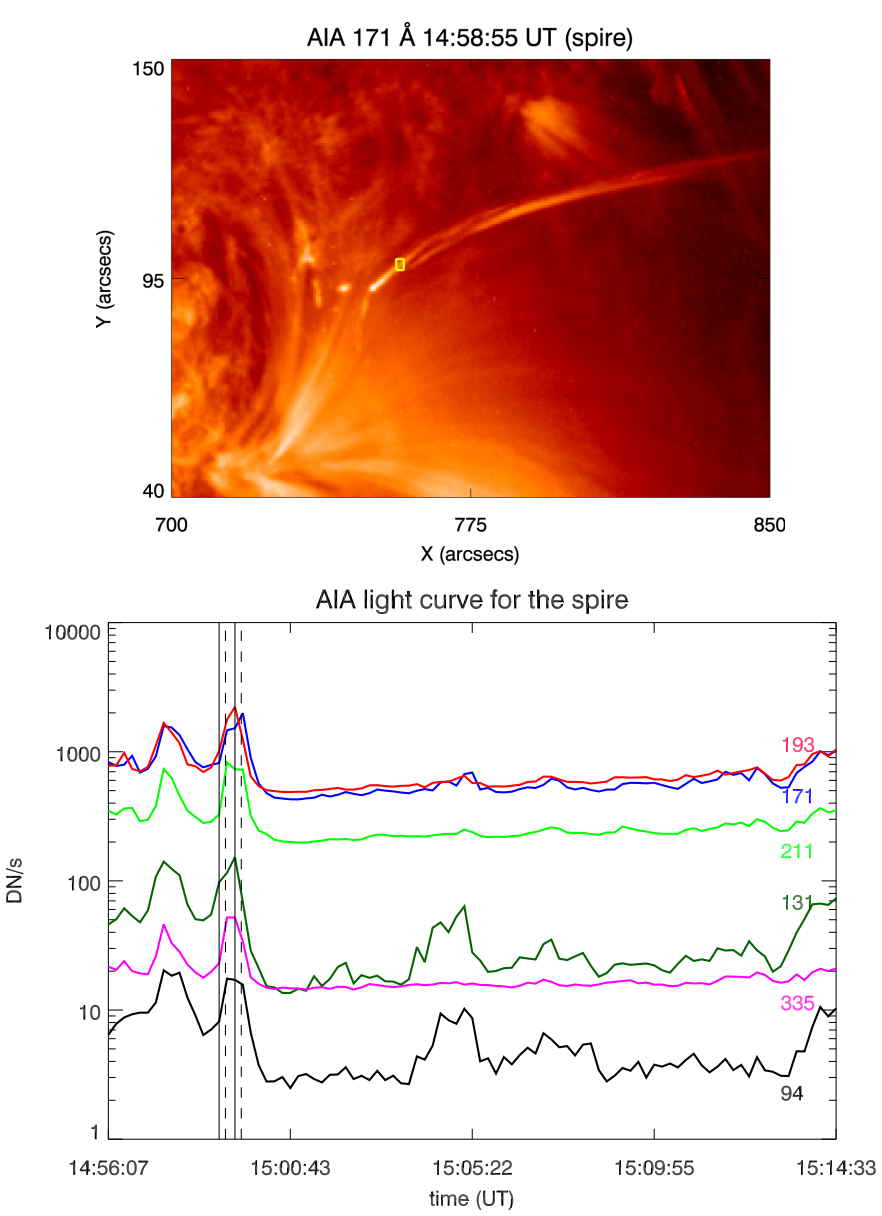

Fig. 4. Top panel: AIA $171 \AA$ image at 14:58 UT. The overplotted yellow box indicates the region of the jet spire selected for further analysis shown as a small box in Fig. 6 (top panel - first column). Bottom panel: temporal evolution of the jet spire calculated in the same small box using all AIA coronal channels. The overplotted solid lines indicate the EIS slit position start time for two exposures in which EIS observed the spire and dashed lines indicate the slit position end times.

observations. Also, we discuss DEM results obtained by combining the AIA and the XRT imaging observations.

\subsubsection{EIS DEM}

We used a set of EIS lines (see Table 3) emitted by various elements over a wide range of temperatures $(\log T[\mathrm{~K}]=5.7$ to $\log$ $T[\mathrm{~K}]=6.4$ ) to calculate the line-of-sight DEM. Emission for the jet spire and the jet footpoint covered two consecutive slit positions, $1^{\prime \prime}$ wide, during 14:58:55-14:59:17 UT (exposure number -9 and 10) and 16:35:08-16:35:30 UT (exposure number 20 and 21), respectively.

We have extracted a small region of $2^{\prime \prime} \times 3^{\prime \prime}(2 \times 3$ pixels $)$ area around the jet spire and footpoint, which are shown as small white boxes in Fe VIII raster image in Fig. 6 (first column). We then obtained an EIS averaged spectrum for these regions and performed a DEM analysis on the EIS intensities (see Table 3) using the XRT_DEM_ITERATIVE2 method (Weber et al. 2004), which is available within SSW. We used IDL routine CHIANTI_DEM to calculate the contribution function $C\left(T, \lambda_{i j}, N_{\mathrm{e}}\right)$ at a constant electron density. We used the electron density calculated from the Fe XII lines intensity ratio technique (see Sect. 3.4) and a set of photospheric abundances by Asplund et al. (2009) in this calculation.
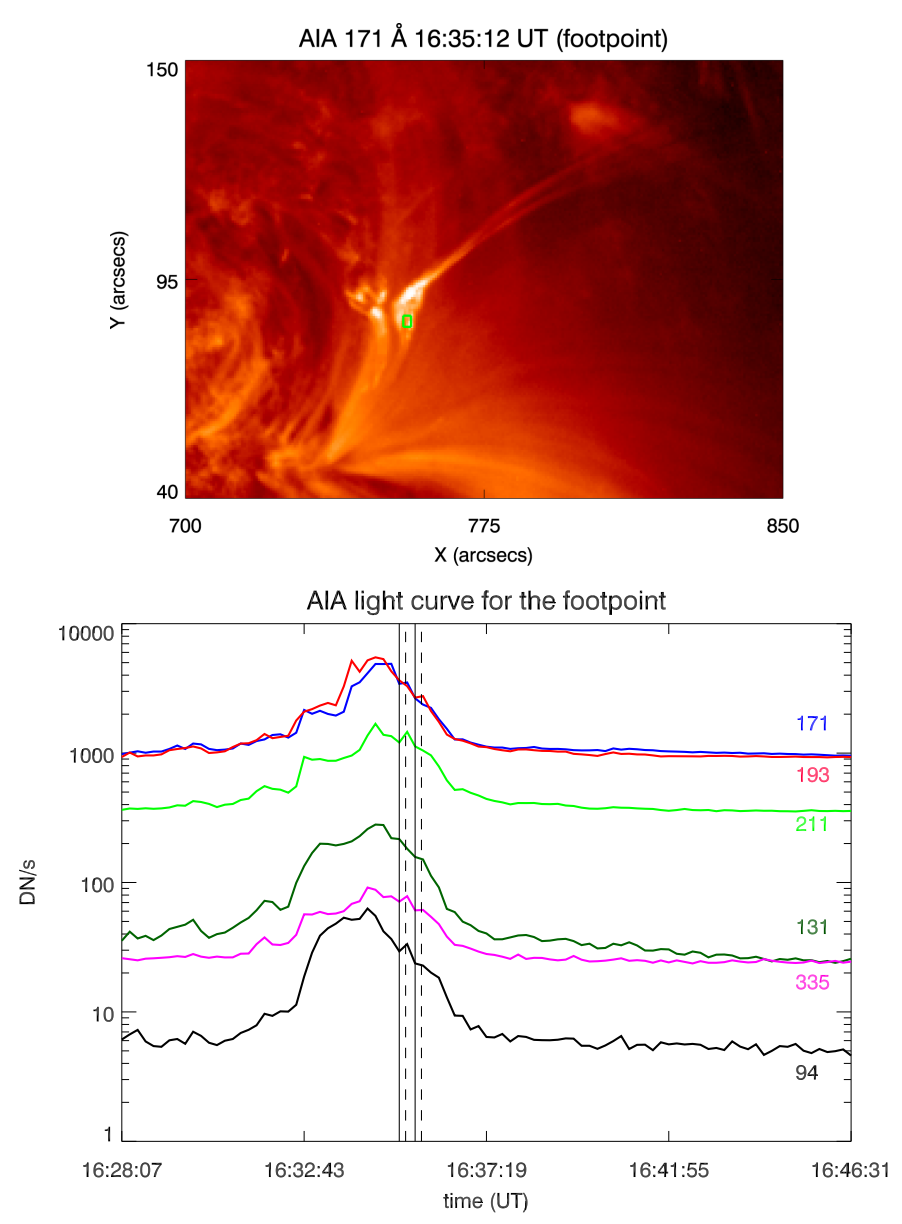

Fig. 5. Same as Fig. 4, for the region of the jet footpoint (shown as a small box in Fig. 6; bottom panel, first column).

Figure 9 shows the DEM curves for the jet spire (top panel) and jet footpoint (bottom panel) obtained from the EIS data in the temperature range between $\log T[\mathrm{~K}]=5.5$ and 7.1 with an interval of $\log T[\mathrm{~K}]=0.05$. The DEM for the jet-spire peaks at $\log T[\mathrm{~K}]=6.3$ with $\mathrm{DEM}=2.1 \times 10^{22} \mathrm{~cm}^{-5} \mathrm{~K}^{-1}$, and for the jet footpoint; it also peaks at $\log T[\mathrm{~K}]=6.3$ with $\mathrm{DEM}=3.9 \times 10^{22} \mathrm{~cm}^{-5} \mathrm{~K}^{-1}$. In order to estimate uncertainties on the DEM, we computed Monte Carlo (MC) realizations of the data. We considered a $20 \%$ uncertainty in the intensities, which takes uncertainties in the atomic data into account (Del Zanna 2013a), and we obtained 400 MC solutions by randomly varying the input intensities. We applied similar methodology given by Cheng et al. (2012) to plot the MC solutions on the best-fit DEM solution. The blue rectangles represents $50 \%$, red rectangles $80 \%$, and yellow rectangles $95 \%$ of the MC solutions in each temperature bin (See Fig. 9).

The EIS DEM curves are not very well constrained above $\log T[\mathrm{~K}]=6.4$, since the highest temperature line observed was Fe XV (1284.160). No emission was observed from Fe XVII $(\lambda 254.87)$ at $\log T[\mathrm{~K}]=6.75$. In the high temperature range ( $>\log T=6.5$ ), the DEM falls sharply. In the lower temperature range, there are no lines observed between $\log T[\mathrm{~K}]=5.8$ and $\log T[\mathrm{~K}]=6.1$ in the EIS observing sequence used, so the DEM values in this range are less reliable.

\subsubsection{AIA-XRT DEM}

Using the co-aligned XRT images from the Ti-poly filter together with time-averaged AIA images in six wavelength 
S. M. Mulay et al.: Temperature and density structure of a recurring active region jet

Table 3. List of the EIS observed emission lines used to constrain DEM curves.

\begin{tabular}{ccccccc}
\hline \hline $\begin{array}{c}\text { EIS observed lines } \\
\text { used for the DEM }\end{array}$ & $\begin{array}{c}\lambda \\
(\AA)\end{array}$ & $\begin{array}{c}\log T_{\max } \\
(\mathrm{K})\end{array}$ & $\begin{array}{c}\text { Observed } \\
\text { (spire) }\end{array}$ & $\begin{array}{c}\text { Predicted } \\
(\text { spire })\end{array}$ & $\begin{array}{c}\text { Observed } \\
\text { (footpoint) }\end{array}$ & $\begin{array}{c}\text { Predicted } \\
\text { (footpoint) }\end{array}$ \\
\hline Fe VIII (bl) & 186.605 & 5.8 & 445 & $562(26 \%)$ & 1617 & $1982(23 \%)$ \\
Fe XII & 195.119 & 6.2 & 1707 & $1755(3 \%)$ & 3270 & $3415(4 \%)$ \\
Si X & 261.056 & 6.2 & 408 & $298(-27 \%)$ & 790 & $629(-20 \%)$ \\
Mg VI & 268.991 & 5.7 & 106 & $62(-42 \%)$ & 355 & $240(-32 \%)$ \\
Fe XV (bl) & 284.160 & 6.4 & 9221 & $9943(8 \%)$ & 16270 & $17170(6 \%)$ \\
\hline
\end{tabular}

Notes. Column 4 and 5 represents observed and predicted intensities (obtained from the EIS DEM) for the jet-spire region; Cols. 6 and 7 represents observed and predicted intensities (obtained from the EIS DEM) for the jet-footpoint region; EIS intensities are in $\mathrm{erg} \mathrm{cm}^{-2} \mathrm{~s}^{-1} \mathrm{sr}^{-1}$ units, bl blended lines; and percentage difference between observed and predicted intensities are given in parenthesis in Cols. 5 and 7.
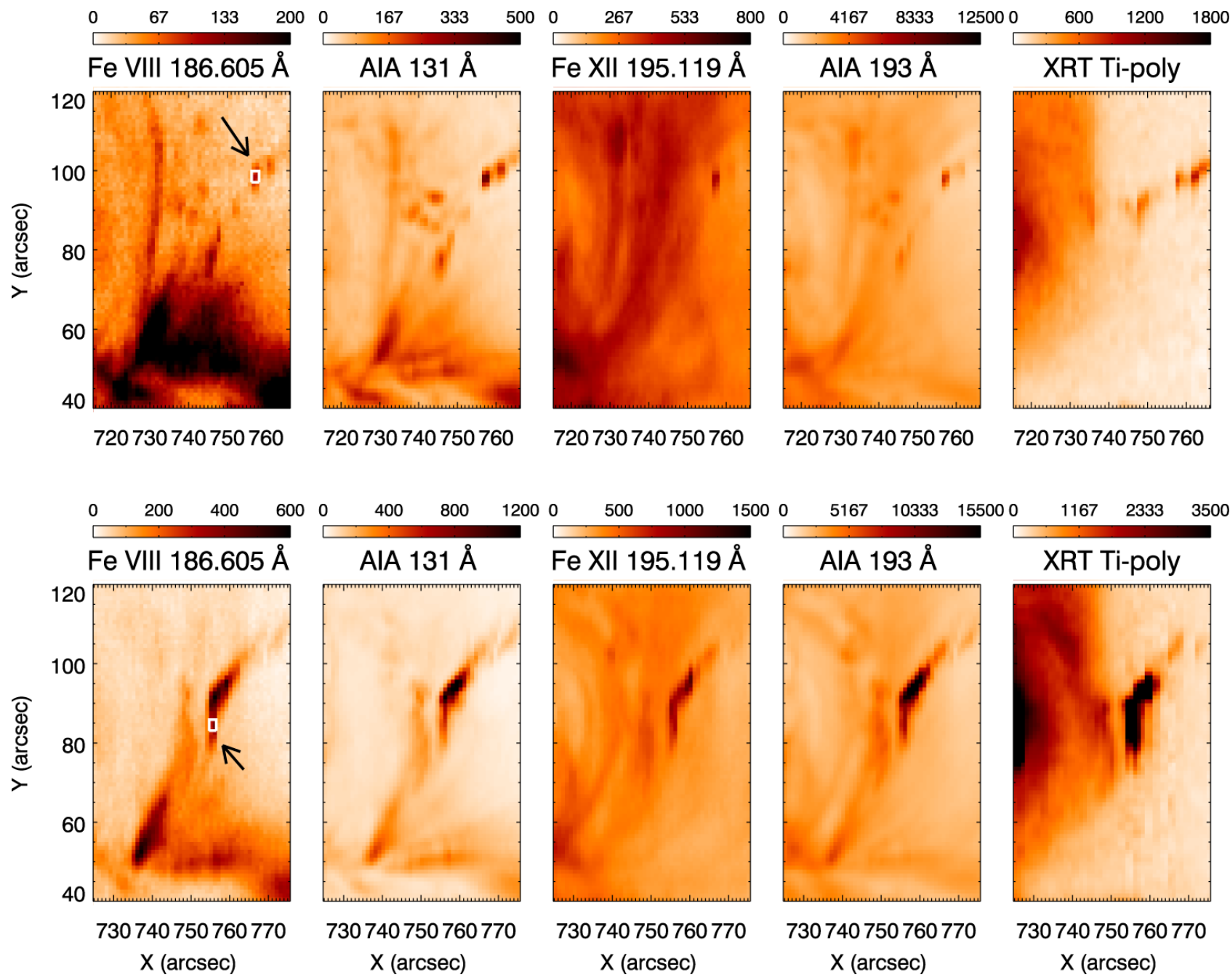

Fig. 6. AIA-EIS-XRT co-aligned images (reverse colour) of the jet spire (top panel) and the jet footpoint (bottom panel). Column 1 and 3: EIS

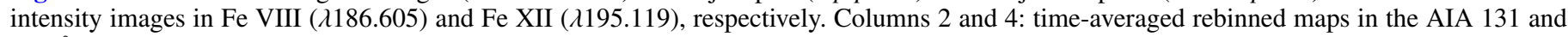
$193 \AA$ A channels, respectively. Column 5: a coaligned map in the Ti-poly filter. The colour bars for the EIS rasters indicate the actual calibrated units (phot $\mathrm{cm}^{-2} \mathrm{~s}^{-1} \operatorname{arcsec}^{-2}$ ) and the units are DN s${ }^{-1}$ EIS pixel ${ }^{-1}$ for AIA images. The regions shown with white boxes (indicated by black arrows) are used for the DEM analysis (see Sect. 3.6).

channels $(94,131,171,193,211$, and $335 \AA$ A), which are sensitive to a range of coronal temperatures, we performed the DEM analysis again using the same method described in Sect. 3.6.1. The purpose of using X-ray Ti-poly images in the DEM analysis is to constrain high temperature emission.

We extracted the same small region of $2^{\prime \prime} \times 3^{\prime \prime}(2 \times 3$ pixels $)$ area around the jet spire and the footpoint, which we used to obtained DEM curves from the EIS. The DN per sec per EIS pixel in each of the six time-averaged AIA channels and the Ti-poly filter were spatially averaged over all pixels in the region. We used these count rates as an input to compute DEM. For the inversion, we used a range of temperatures $\log T[\mathrm{~K}]=5.5$ to $\log T[\mathrm{~K}]=7.1$ with $\triangle \log T=0.05$ intervals.

The make_xrt_wave_resp.pro routine was used to produce the spectral responses for the Ti-poly filter. We obtained XRT and AIA temperature responses from the CHIANTI v.8 (Del Zanna et al. 2015) atomic database using the ISOTHERMAL procedure (see Appendix in Del Zanna et al. 2011). We 

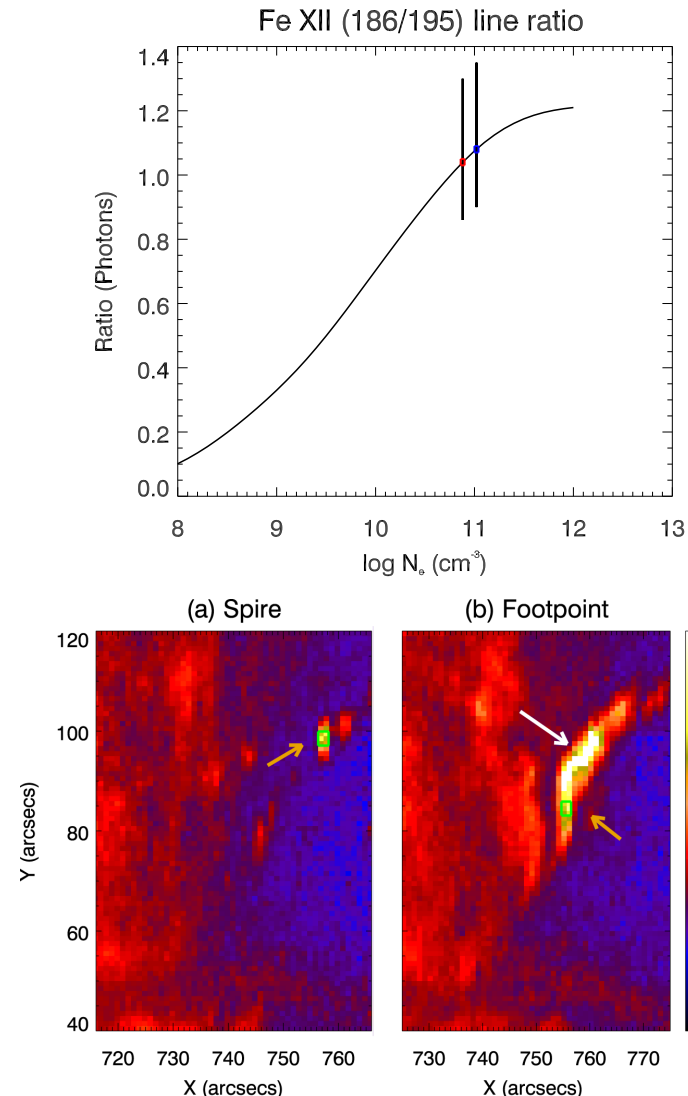

(b) Footpoint

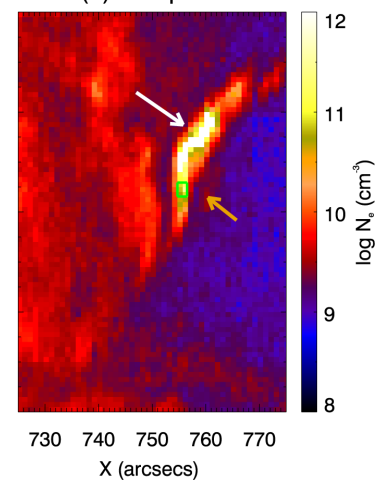

Fig. 7. Top panel: theoretical intensity of the Fe XII $(\lambda 186 / \lambda 195)$ line ratio as a function of electron density. The density ratio for the jet spire and jet footpoint are shown with red and blue boxes, respectively. The vertical lines overplotted on the curve shows the errors estimated by considering $20 \%$ uncertainty. Bottom panels: electron density maps for the a) jet spire and the b) jet footpoint. The electron densities are in logarithmic scale in $\mathrm{cm}^{-3}$ units. The regions used for the density diagnostics are shown with green boxes (indicated by orange arrows).

calculated the AIA responses per EIS pixel to compare the DEM results from the EIS with those obtained by the AIA-XRT instruments (see Figs. A.1 and A.2 in the Appendix). We took into account the time-dependent degradation factor for these instruments as in the SSW. We used a set of photospheric abundances (Asplund et al. 2009) along with the electron density obtained from the Fe XII line intensity ratio (see Sect. 3.4) in this calculation.

Figure 10 shows the DEM curves (black curves) for the jet spire (top panel) and the jet footpoint (bottom panel), respectively. The errors on the DEM curves were estimated by considering a $20 \%$ uncertainty in the intensities, coming primarily from the atomic data. The blue rectangles represents $50 \%$, red rectangles $80 \%$, and yellow rectangles $95 \%$ of the MC solutions in each temperature bin. The DEM for the jetspire peaks at $\log T[\mathrm{~K}]=6.3$ with $\mathrm{DEM}=2.1 \times 10^{22} \mathrm{~cm}^{-5} \mathrm{~K}^{-1}$, and for the jet footpoint, and it also peaks at $\log T[\mathrm{~K}]=6.3$ with $\mathrm{DEM}=3.4 \times 10^{22} \mathrm{~cm}^{-5} \mathrm{~K}^{-1}$. The error bars below $\log T$ $[\mathrm{K}]=5.9$ and above $\log T[\mathrm{~K}]=6.4$ are large, so the DEM values in this range are less reliable. Table 4 shows the observed (Cols. 2 and 5) and predicted (Cols. 3, 4 and 6, 7) intensities (average DN/s per EIS pixel) in the region of the jet spire and the jet footpoint, respectively. The intensities reproduced by the AIAXRT DEM are closer to the observed intensities than the intensities reproduced by the EIS DEM. In the case of the AIA $94 \AA$ channel, the EIS DEM shows large differences in the observed
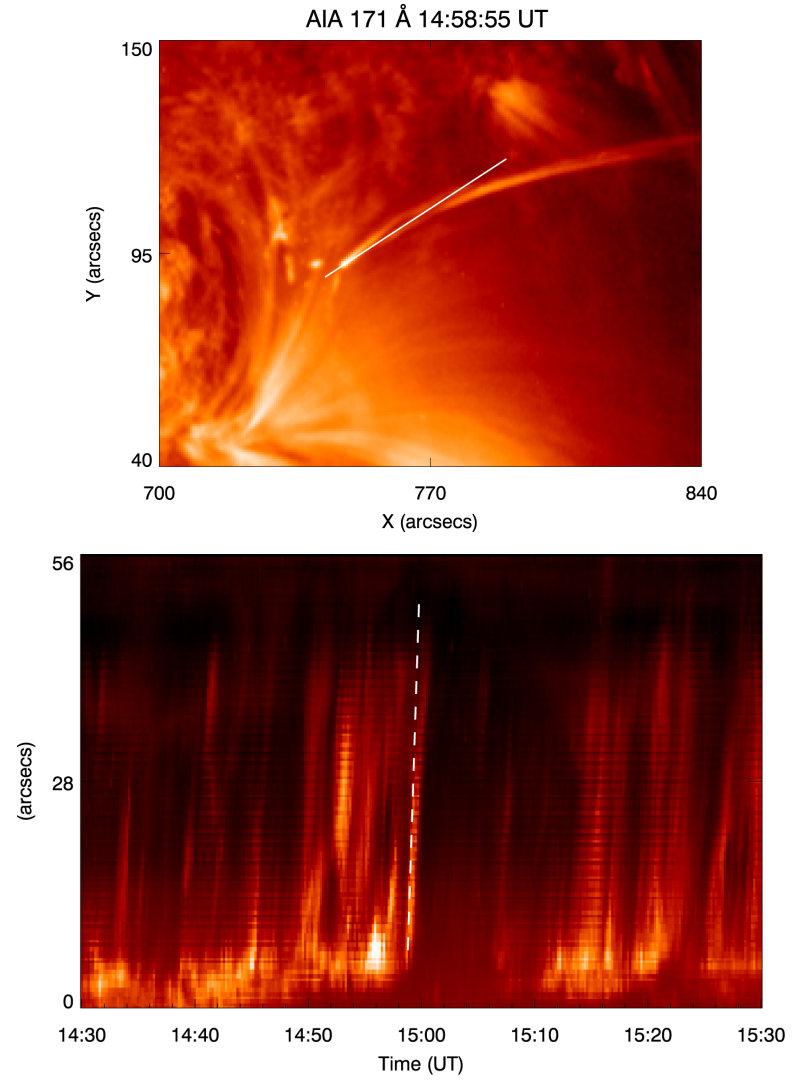

Fig. 8. Top panel: the jet evolution in the AIA $171 \AA$ channel at 14:58:55 UT. The overplotted white line shows an artificial slit, which is used to produce a time-distance plot. Bottom panel: time-distance plot along the jet spire for an hour from 14:30 to 15:30 UT. The white dashed line used for the velocity calculation, which is found to be $524 \mathrm{~km} \mathrm{~s}$.

and predicted intensities. Since the EIS observation only covers temperature between $\log T[\mathrm{~K}]=5.7$ and 6.4 , it is possible that the EIS DEM is unable to reproduce the high temperature emission that is observed by the AIA and XRT. Although the peak DEM values and temperature of peak DEM are similar for both the EIS and AIA-XRT DEMs, the high temperature values $(\log T[\mathrm{~K}]>6.5)$ for AIA-XRT do not fall as sharply as for EIS, although the scatter of MCMC results is large.

\subsection{Emission measure and filling factor measurement}

The emission measure can be measured from individual spectral emission lines at their temperature of formation (Pottasch 1963). Considering a spectral line formed over a range of temperatures, $\log T_{\max }-0.15$ to $\log T_{\max }+0.15$ (where $T_{\max }$ is the temperature where the contribution function has its maximum), we can determine the emission measure by integrating the DEM values within that temperature range.

In this analysis, we consider the Fe XII line formed over a temperature range from $\log T[\mathrm{~K}]-6.05$ to 6.35 and we calculated emission measure from the EIS DEM and also from the AIA-XRT DEM in the region of the spire and the footpoint.

The column depth for the spire and the footpoint is estimated from the size of the observed structure in the EIS raster images. In this study, we observed the emission from the spire and the footpoint over two consecutive EIS raster positions, i.e. for $2^{\prime \prime}$. By assuming that the structure has a cylindrical geometry, we calculated a filling factor using Eq. (5). The filling factor 
S. M. Mulay et al.: Temperature and density structure of a recurring active region jet

Table 4. Observed and predicted SDO/AIA count rates for the jet spire and jet footpoint obtained using photospheric abundances.

\begin{tabular}{|c|c|c|c|c|c|c|}
\hline $\begin{array}{c}\text { Band } \\
(\AA)\end{array}$ & $\begin{array}{c}\text { Observed } \\
\text { spire }\end{array}$ & $\begin{array}{c}\text { Predicted } \\
\text { (AIA-XRT DEM) } \\
\text { spire }\end{array}$ & $\begin{array}{l}\text { Predicted } \\
\text { (EIS DEM) } \\
\text { spire }\end{array}$ & $\begin{array}{l}\text { Observed } \\
\text { footpoint }\end{array}$ & $\begin{array}{c}\text { Predicted } \\
\text { (AIA-XRT DEM) } \\
\text { footpoint }\end{array}$ & $\begin{array}{l}\text { Predicted } \\
\text { (EIS DEM) } \\
\text { footpoint }\end{array}$ \\
\hline AIA 94 & 45 & $27(-40 \%)$ & $10(-78 \%)$ & 75 & $67(-11 \%)$ & $22(-71 \%)$ \\
\hline AIA 131 & 303 & $244(-20 \%)$ & $112(-63 \%)$ & 455 & $350(-23 \%)$ & $366(-20 \%)$ \\
\hline AIA 171 & 4747 & $5930(25 \%)$ & $2134(-55 \%)$ & 7651 & $7565(-1 \%)$ & $5867(-23 \%)$ \\
\hline AIA 193 & 4494 & $5144(15 \%)$ & $3181(-29 \%)$ & 8063 & $7558(-6 \%)$ & $6942(-14 \%)$ \\
\hline AIA 211 & 1946 & $1984(2 \%)$ & $1461(-25 \%)$ & 2957 & $2976(1 \%)$ & $2891(-2 \%)$ \\
\hline AIA 335 & 117 & $101(14 \%)$ & $59(-50 \%)$ & 179 & $170(-5 \%)$ & $116(-35 \%)$ \\
\hline
\end{tabular}

Notes. Columns 2 and 5 indicate the observed count rates (averaged DN/s per EIS pixel) for the region of the jet spire and the jet footpoint, respectively. Column 3,4 and 6, 7 indicate predicted count rates obtained from the AIA-XRT DEM and EIS DEM for the region of the jet spire and the jet footpoint, respectively. Values in parenthesis in Cols. 3, 4 and 6, 7 show the percentage differences between observed and predicted intensities.

Table 5. Plasma parameters calculated using EIS data and DEM curves.

\begin{tabular}{|c|c|c|c|c|}
\hline \multirow[t]{2}{*}{ Event } & \multicolumn{2}{|c|}{ Photospheric abundances } & \multicolumn{2}{|c|}{ Coronal abundances } \\
\hline & AIA-XRT DEM & EIS DEM & AIA-XRT DEM & EIS DEM \\
\hline \multicolumn{5}{|l|}{ Jet-spire } \\
\hline $\log T(\mathrm{~K})$ for peak DEM & 6.3 & 6.3 & 6.3 & 6.3 \\
\hline Peak DEM $\left(\mathrm{cm}^{-5} \mathrm{~K}^{-1}\right)$ & $2.1 \times 10^{22}$ & $2.1 \times 10^{22}$ & $5.3 \times 10^{21}$ & $5.5 \times 10^{21}$ \\
\hline $\mathrm{EM}$ for Fe XII $\left(\mathrm{cm}^{-5}\right)$ & $2.2 \times 10^{28}$ & $1.7 \times 10^{28}$ & $5.4 \times 10^{27}$ & $4.3 \times 10^{27}$ \\
\hline Width of the spire $(\mathrm{cm})$ & & $1.5 \times 10^{8}$ & & \\
\hline Density from Fe XII $\left(\mathrm{cm}^{-3}\right)$ & & $7.6 \times 10^{10}$ & & \\
\hline Filling factor from EIS & & 0.02 & & 0.005 \\
\hline \multicolumn{5}{|l|}{ Jet-footpoint } \\
\hline $\log T(\mathrm{~K})$ for peak DEM & 6.3 & 6.3 & 6.3 & 6.3 \\
\hline Peak DEM $\left(\mathrm{cm}^{-5} \mathrm{~K}^{-1}\right)$ & $3.4 \times 10^{22}$ & $3.9 \times 10^{22}$ & $9.5 \times 10^{21}$ & $1.0 \times 10^{22}$ \\
\hline EM for Fe XII $\left(\mathrm{cm}^{-5}\right)$ & $3.5 \times 10^{28}$ & $3.4 \times 10^{28}$ & $9.6 \times 10^{27}$ & $8.6 \times 10^{27}$ \\
\hline Width of the footpoint $(\mathrm{cm})$ & & $1.5 \times 10^{8}$ & & \\
\hline Density from Fe XII $\left(\mathrm{cm}^{-3}\right)$ & & $1.1 \times 10^{11}$ & & \\
\hline Filling factor from EIS & & 0.02 & & 0.005 \\
\hline
\end{tabular}

was 0.02 in both regions using photospheric abundances and 0.005 for coronal abundances.

Table 5 shows the results for plasma parameters. The values obtained from AIA-XRT DEM are given in Cols. 2 and 4 and the values obtained from the EIS DEM curves are given in Cols. 3 and 5, respectively.

\subsection{Synthetic spectrum}

Synthetic spectra were calculated from the EIS DEM and AIAXRT DEM curves in the region of the spire and the footpoint. The purpose was to see whether the DEM curves predict any high temperature emission, such as emission from Fe XVII

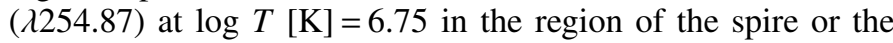
footpoint (even though EIS did not show any evidence of this).

We used the CH_SS CHIANTI routine to obtain the spectra. We calculated synthetic spectra in the SW and LW channels of the EIS instrument using the same set of parameters (abundances and densities; see Table 5). We adopted the radiometric calibration by Del Zanna (2013a) to calculate the EIS effective areas. The synthetic spectra were then convolved with the effective areas of both channels and the contribution of spectral lines and continuum emission was determined (see the Appendix for details).

The synthetic spectra obtained from the EIS DEM and AIAXRT DEM curves predict similar lines. Small differences were seen in the intensities, which could be explained by the highly variable nature of the jet structure. The AIA light curves in these regions (see Figs. 4 and 5) show changes in the count rates in all AIA channels during the EIS raster observations. Also, we have to take into account the spatial resolution and exposure times for EIS, AIA, and XRT instruments, which also has an effect on the DEM curves.

The SW and LW channels show more count rates in the case of the jet footpoint compared to the spire structure. The Fe XII ( $\lambda 195.119)$ line dominates the SW channel; whereas Fe XIV

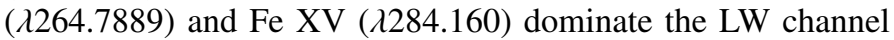
in the synthetic spectra. Neither synthetic spectra for the spire predict any measurable contribution from the high temperature lines, such as Fe XVII ( 2254.87$)$ at $\log T[\mathrm{~K}]=6.75$, confirming that the high temperature part of the DEM is consistent with the observed EIS spectra. In the case of the footpoint region, both synthetic spectra predict weak contributions from Ca XVII

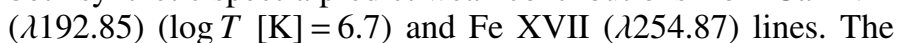
predicted Fe XVII ( $\lambda 254.87)$ signal is weak but inconsistent with the EIS observation, which shows no signal.

\subsection{Effect of varying the temperature range and abundances in the DEM analyses}

We investigated the effects of varying the temperature range and elemental abundances in the DEM analyses. We followed the same procedure given in Sect. 3.6 to obtained DEM from AIA-XRT and the EIS observations using coronal abundances by Feldman (1992) and a temperature range from $\log T[\mathrm{~K}]=5.5$ to 7.1. We obtained similar curves (in shape) as shown in Figs. 9 

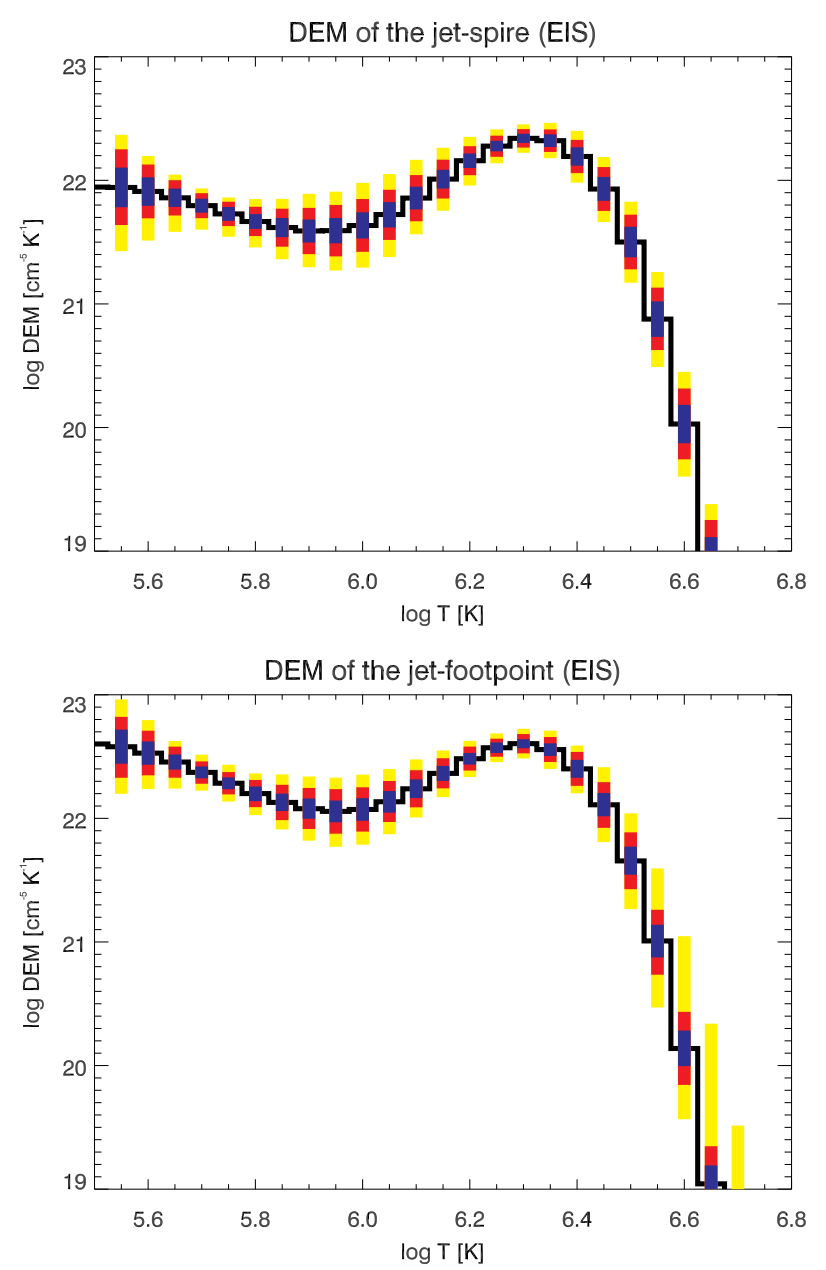

Fig. 9. EIS DEM curves for the jet spire (top panel) and the jet footpoint (bottom panel). The DEMs are obtained from the XRT_DEM_ITERATIVE2 method (black curve). The blue rectangles represent $50 \%$, red rectangles $80 \%$, and yellow rectangles $95 \%$ of the MC solutions in each temperature bin.

and 10. The jet spire and the jet footpoint showed the same peak temperature (see Table 5). However, the peak DEM, EM, and filling factor values obtained using the coronal abundances were found to be a factor of four lower than the values obtained using the photospheric abundances.

\subsection{AlA count rates}

The EIS DEM curves were used to predict the total count rates in each AIA channel. We used the CH_SS CHIANTI routine to obtain synthetic spectra in each of the AIA channel. The effective areas of each AIA channel were calculated per EIS pixel. We examined the contribution of spectral lines and continuum emission to each of the channel. The results confirm the multithermal emission contributing to the AIA channels in both regions, where the only exception is the AIA $171 \AA$ channel.

Figures 11 and 12 shows the predicted total count rates in each AIA channel obtained from the EIS DEM and AIA-XRT DEM curves for the region of the spire and region of the footpoint, respectively. The differences seen in the observed and predicted count rates can be attributed to the variability seen in the jet structure (see Figs. 4 and 5). Count rates in each AIA channel
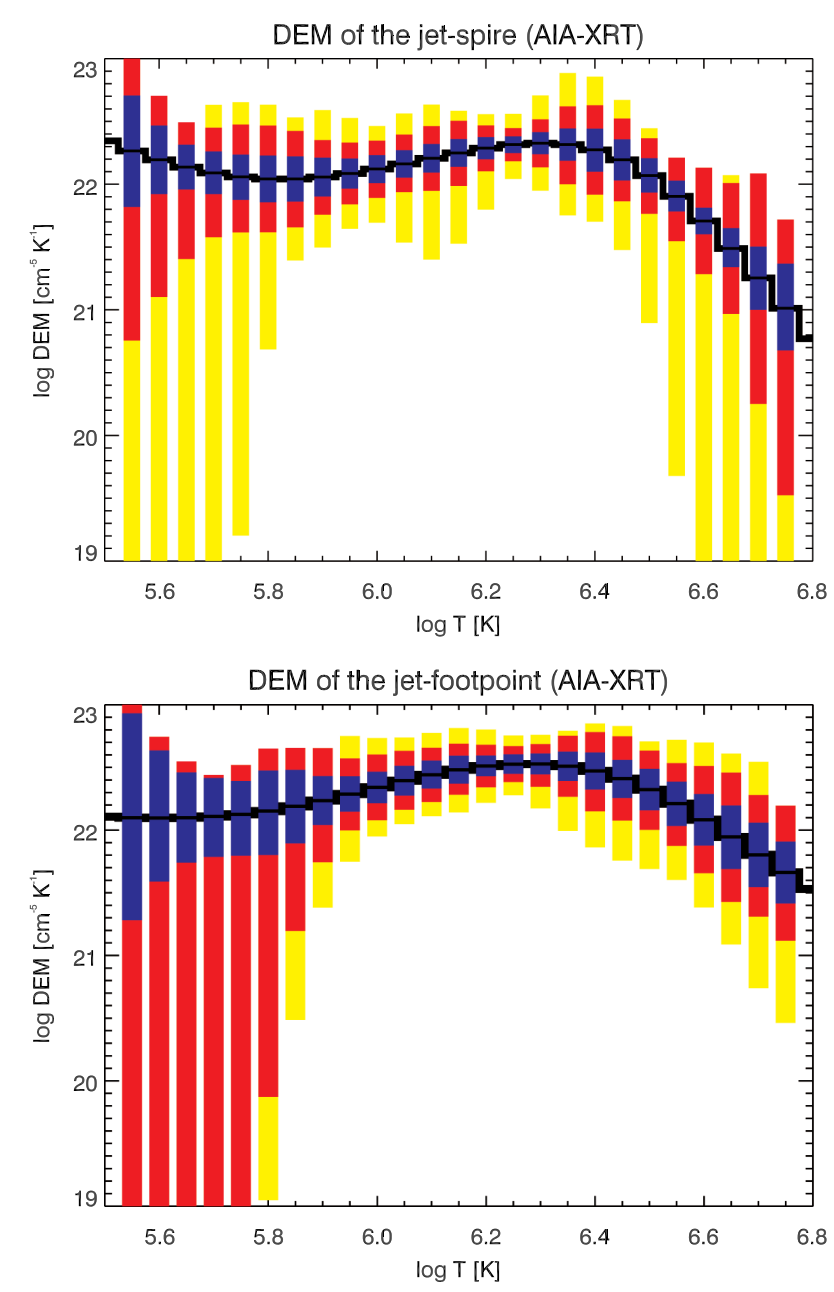

Fig. 10. AIA-XRT DEM curves for the jet spire (top panel) and jet footpoint (bottom panel). The DEMs are obtained from the XRT_DEM_ITERATIVE2 method (black curve). The blue rectangles represent $50 \%$, red rectangles $80 \%$, and yellow rectangles $95 \%$ of the MC solutions in each temperature bin.

are found to be higher in the region of the footpoint than in the region of the spire (see Table 4).

\subsection{The evolution of the footpoint}

The continuous coverage of the jet with SDO/AIA and Hinode/XRT enabled us to investigate the temporal evolution of the footpoint region further. Figure 5 (bottom panel) shows the temporal evolution of the footpoint region in all AIA coronal channels and its variable nature is clearly seen over the period of time. Before the EIS raster times (16:35:08 and 16:35:30 UT; shown as black lines in Fig. 5 bottom panel), the light curves showed a peak in the AIA $94 \AA$ and $335 \AA$ channels at 16:34 UT. These peaks were followed by peaks in the $211 \AA$, the $193 \AA$ and, finally, the 171 and $131 \AA$ emission. This scenario indicates that the hot temperature cooled down over the period of time from $\log T[\mathrm{~K}]=6.6$ and that EIS observed the jet footpoint during its cooling phase.

In order to investigate this further, we performed a DEM analysis using the AIA and XRT images. We took the AIA images $\left(0.6^{\prime \prime}\right.$ per pixel), which are closest to the XRT timings, and degraded them to the XRT resolution (1.02"). We performed the DEM analysis for every 1 min of observations from 16:30 UT to 
Table 6. Estimated and predicted Fe XVIII count rates (averaged DN/s) for the AIA $94 \AA$ channel obtained from the AIA-XRT DEM (see Fig. 14) using the photospheric abundances for the jet-footpoint region shown in Fig. 13.

\begin{tabular}{ccc}
\hline \hline $\begin{array}{c}\text { Time } \\
(\mathrm{UT})\end{array}$ & $\begin{array}{c}\text { Estimated } \\
\text { (from AIA 94 A) }\end{array}$ & $\begin{array}{c}\text { Predicted } \\
\text { (from DEM) }\end{array}$ \\
\hline 16:30:38 & 0.4 & 3 \\
16:32:50 & 17 & 20 \\
16:34:14 & 34 & 28 \\
16:35:02 & 12 & 18 \\
\hline
\end{tabular}

16:40 UT in the same box at the region of the footpoint (shown in the Fig. 5, top panel). Figure 13 shows the DEM curves at four different times during the evolution of the footpoint. We observed significant differences in the DEMs for the temperature range from $6.5<\log T[\mathrm{~K}]<6.9$. At 16:30 UT (top left panel); the DEM peaks at $\log T[\mathrm{~K}]=6.4$ and falls sharply until $\log$ $T[\mathrm{~K}]=6.9$. At $16: 32 \mathrm{UT}$ (top right panel), and the DEM also peaks at $\log T[\mathrm{~K}]=6.4$ but the DEM values are increased for the temperatures $>\log T[\mathrm{~K}]=6.6$. The shape of the DEM changes drastically at 16:34 UT (bottom left panel), where it peaks at $\log T[\mathrm{~K}]=6.1$ and gradually decreases until $\log T[\mathrm{~K}]=7.0$. The DEM values at a temperature around $\log T[\mathrm{~K}]=6.7$ has increased rapidly over the period of two minutes. With time, the DEM decreases again for the temperature $>\log T[\mathrm{~K}]=6.5$ at 16:35 UT (bottom right panel). These DEM results indicate that there is a rise in temperature and then a gradual cooling phase following the peak at 16:34 UT. This scenario is consistent with the nature of the observed AIA light curves.

\subsection{Estimation of Fe XVIII emission in the jet footpoint}

During the evolution of the jet footpoint, the DEM curves showed the presence of temperature $>\log T[\mathrm{~K}]=6.7$ (see Fig. 13). We checked whether there is a significant presence of Fe XVIII emission in the footpoint region to investigate the validity of this high temperature. For the AIA $94 \AA$ channel, we obtained Fe XVIII (93.932 $\AA$ ) maps using the empirical combination of the AIA 94, 211, and $171 \AA$ channels given by Del Zanna (2013b). Figure 14 shows the Fe XVIII images at the same timings as those for which we obtained DEMs (see Fig. 13) during the evolution of the footpoint. These images clearly show the Fe XVIII emission in the footpoint region at 16:32, 16:34, and 16:35 UT. The emission from Fe XVIII was not seen at the same small box region at 16:30 UT before the rise in temperature of the footpoint.

We estimated Fe XVIII averaged count rates in the AIA $94 \AA$ channel in the same region (shown with a yellow box in Fig. 14) and compared them with the predicted averaged count rates obtained from the DEM (see Fig. 13). Table 6 shows a very good agreement between the Fe XVIII estimated and predicted count rates at 16:32, 16:34, and 16:35 UT.

\section{Discussion and summary}

In this paper, we present a comprehensive investigation of the temperature structure of the jet spire and the jet footpoint of a recurrent AR jet observed on October 31, 2011 using simultaneous imaging (from the AIA and XRT) and spectroscopic observations (from the EIS instrument). The jets originated from the western edge of AR NOAA 11330 (N08 W49). The highly variable nature of the jet spire and the footpoint was observed during the jet evolution (see Figs. 4 and 5). We also observed plasma blobs moving along the jet spire in the AIA channels (see online Movie 1).

We studied the temperature structure of the jet spire and the jet footpoint by performing a DEM analysis (see Sect. 3.6). The plasma along the line of sight in the jet spire and jet footpoint was found to be peak at $2.0 \mathrm{MK}(\log T[\mathrm{~K}]=6.3)$ and we obtained similar DEM values at the peak of DEM curves (see Table 5). The EIS and AIA-XRT DEM curves in both regions appear to be in good agreement in the temperature interval from $\log T[\mathrm{~K}]=5.9-6.3$. Substantial variations were found between solutions obtained from the MC iterations at lower temperatures. We note that the DEM curves are not well constrained below log $T[\mathrm{~K}]=5.8$ and above $\log T[\mathrm{~K}]=6.4$.

There are various factors that can affect the DEM results, such as the choice of elemental abundances, range of temperatures over which the DEM inversion is performed, uncertainties in the atomic data, and cross-calibration of instruments. We investigated cross-calibration issues by performing a similar analysis, shown in Sect. 3.6 on a moss region (shown as a yellow box in Fig. 1), for which there was very little variation of the intensity with time (see Appendix A). Based on the results obtained from the moss, we have confidence in the calibration of EIS, AIA, and XRT. These results also confirm that the method we used in Sect. 3.6 for the DEM analysis combining AIA and XRT observation is reliable.

The synthetic spectra for the spire do not predict any measurable contribution from the high temperature lines such as Fe XVII ( 2254.87) at $\log T[\mathrm{~K}]=6.75$, confirming that the high temperature part of the DEM is consistent with the observed EIS spectra. In the case of the jet footpoint, both synthetic spectra predict weak contributions from Ca XVII ( 1192.85) and Fe XVII (2254.87). With further investigation, we confirmed that there was emission from the Fe XVIII (93.932 $\AA$ ) lines in the region of the footpoint during the early stages of the jet. We also found a good agreement between the estimated and predicted Fe XVIII count rates. The consistency between the nature of the AIA light curves and changes in the DEM values with temperatures during the evolution of the footpoint and the emission from the Fe XVIII (93.932 ̊) line leads us to conclude that the hot component in the footpoint region was present initially such that the jet had cooled down by the time EIS observed it.

It is important to note that the predicted lines in each AIA channels confirmed the multi-thermal emission contributing to the AIA channels in the region of the spire and the footpoint (see Figs. 11 and 12). This is the first such detailed investigation of this nature of AR jets.

We calculated an electron density using Fe XII $(\lambda 186 / \lambda 195)$ line ratio density diagnostics. The electron density was found to be $N_{\mathrm{e}}=7.6 \times 10^{10} \mathrm{~cm}^{-3}$ at the region of the spire and $1.1 \times 10^{11} \mathrm{~cm}^{-3}$ at the region of the footpoint (see Fig. 7), and taking the $20 \%$ error into account the density could be even higher. For the first time in AR jet studies, we observed a region (shown with a white arrow in Fig. $7 b$, bottom panel), which has Fe XII $\lambda 186 / \lambda 195$ ratio greater than 1.2 . This indicates that the region has a high density $\left(\log N_{\mathrm{e}}>11.5\right)$, which is above the range of sensitivity of Fe XII lines ratio diagnostics.

We also calculated a plasma filling factor using Eq. (5). It was found to be 0.02 in the region of the spire and in the region of the footpoint. These values are in agreement with the values obtained by Chifor et al. (2008b) and Yang et al. (2011) from their 

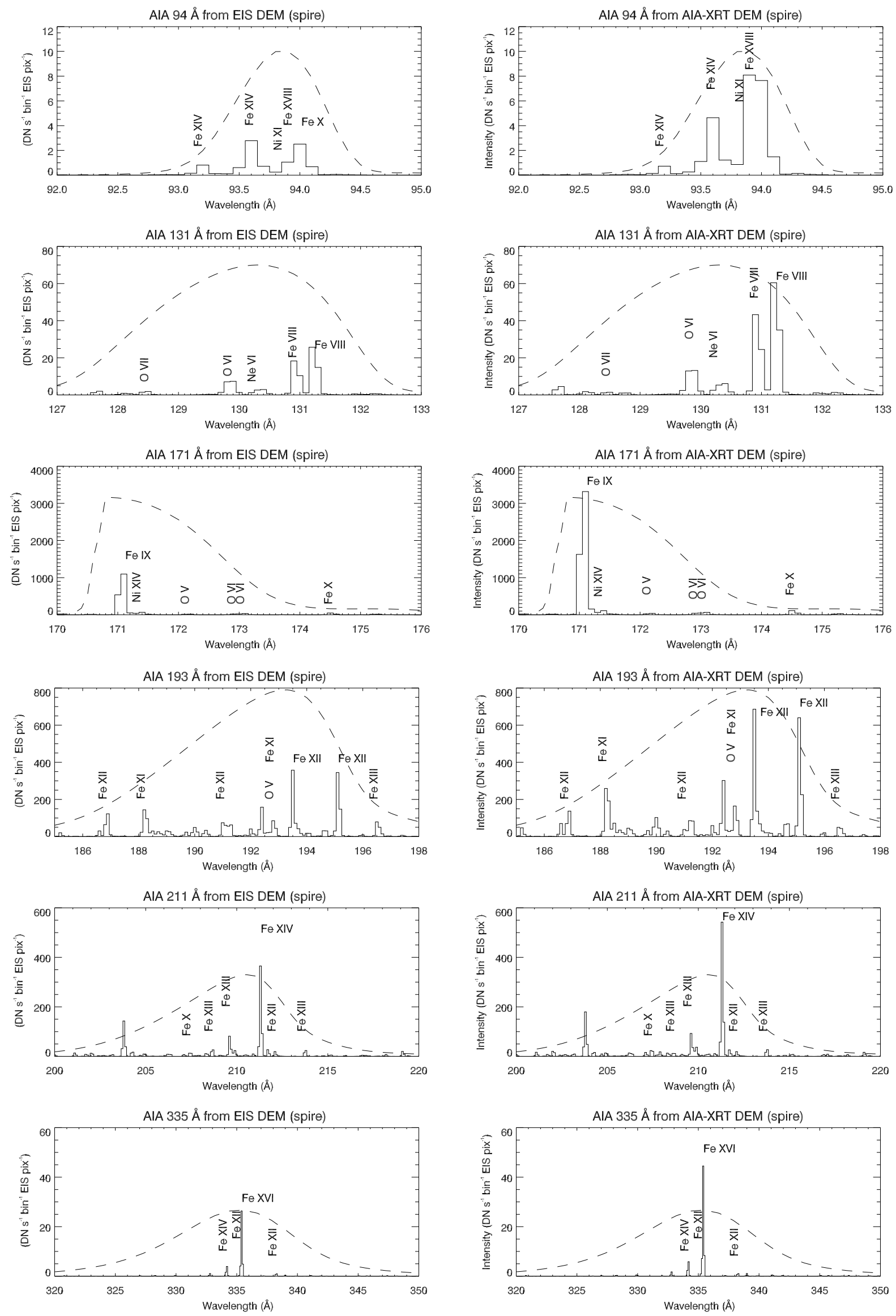

Fig. 11. Synthetic spectra for each AIA channel obtained from the EIS DEM (left panel) and the AIA-XRT DEM (right panel) for the region of the spire. The overplotted dashed lines show the effective area (scaled) of each AIA channels. 
S. M. Mulay et al.: Temperature and density structure of a recurring active region jet
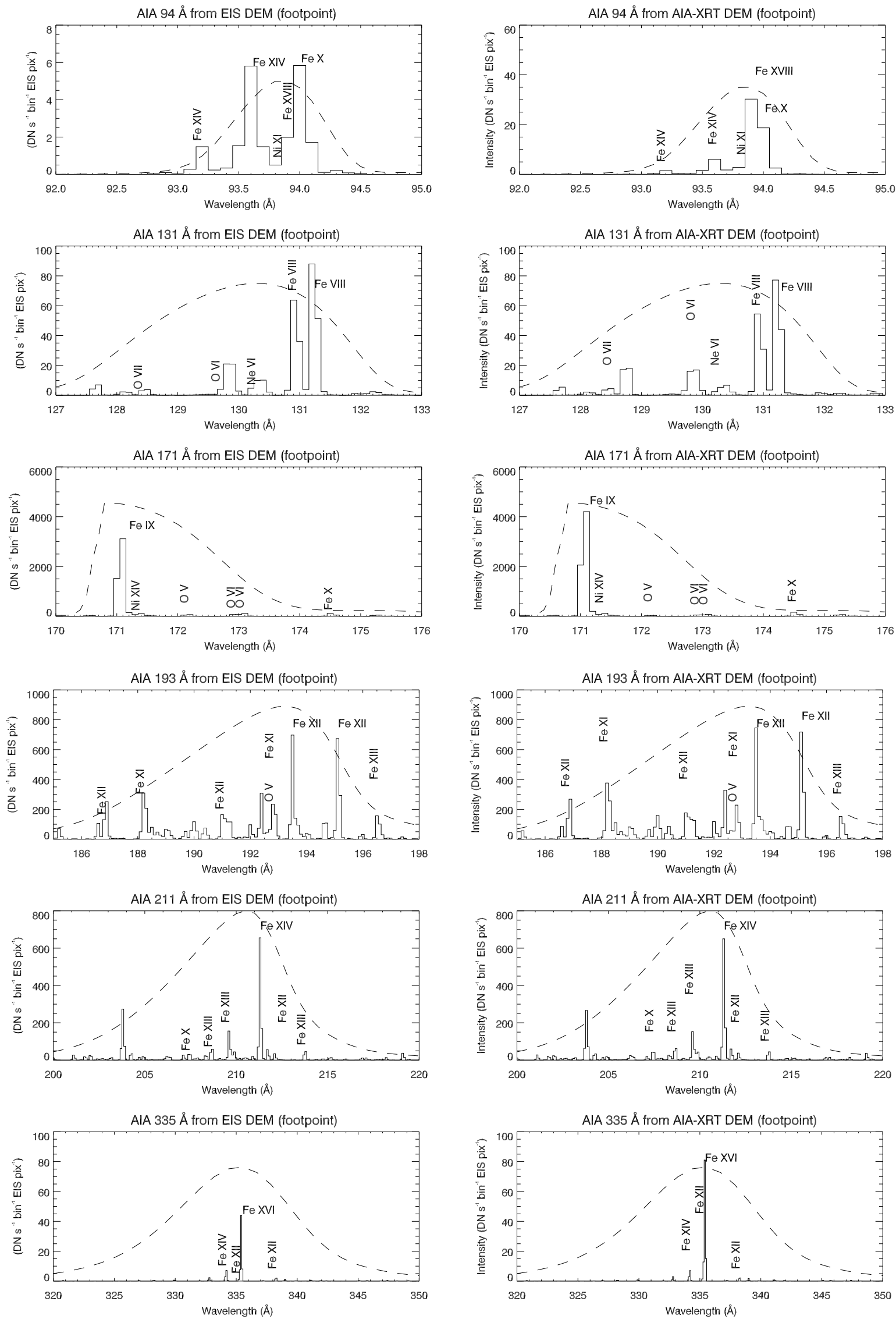

Fig. 12. Synthetic spectra for each AIA channel obtained from the EIS DEM (left panel) and the AIA-XRT DEM (right panel) for the region of the footpoint. The overplotted dashed lines show the effective area (scaled) of each AIA channels. The $Y$-axis scale is different in the case of the AIA $94 \AA$ channel for the intensities predicted from the EIS DEM and AIA-XRT DEM. 

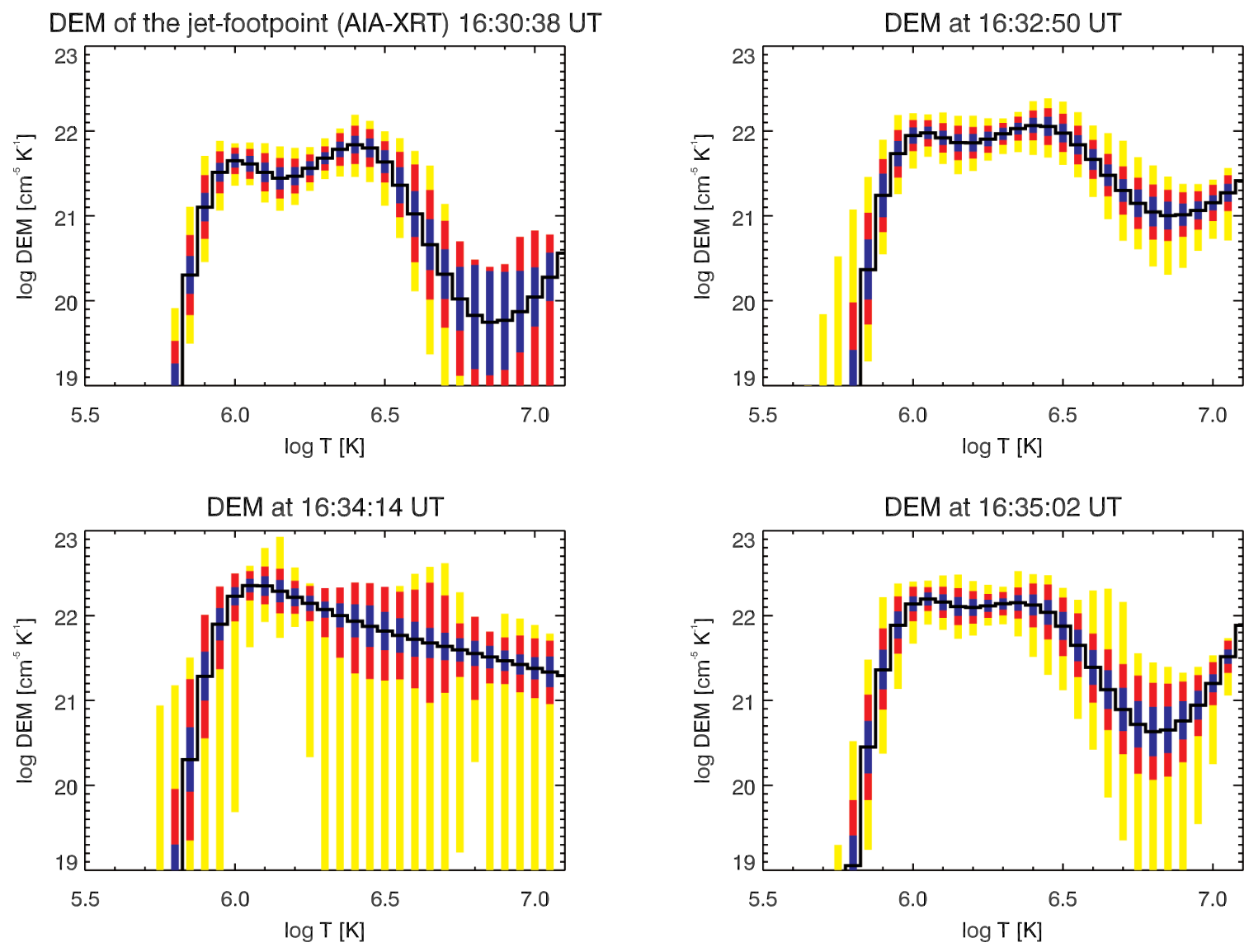

Fig. 13. Results of DEM obtained from the AIA and XRT images during the temporal evolution of the footpoint region.
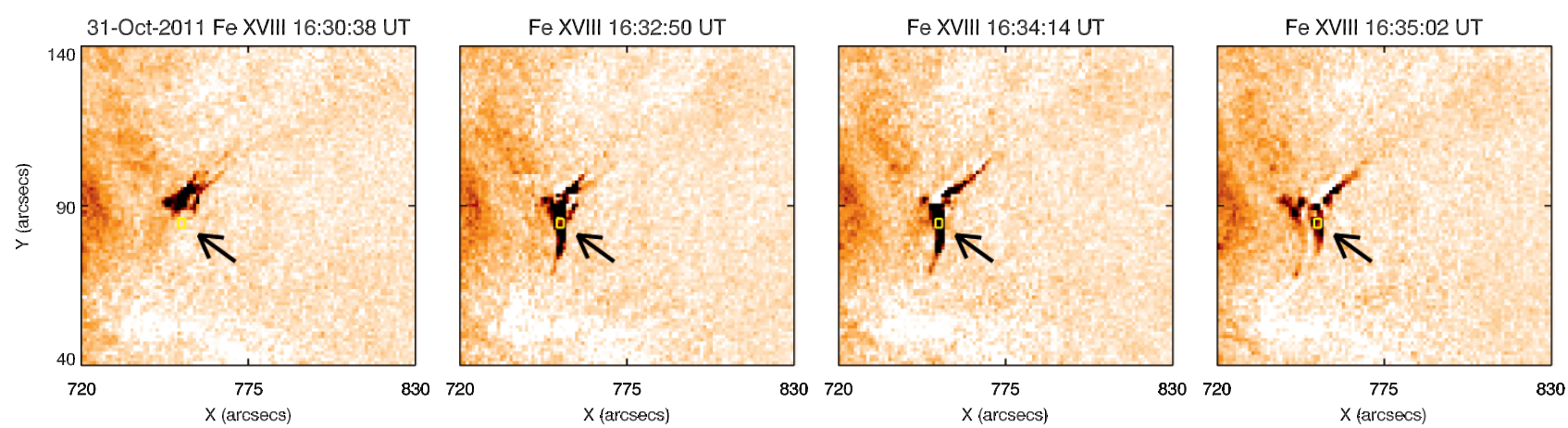

Fig. 14. Maps of Fe XVIII (93.932 Å) during the evolution of the footpoint region. The yellow overplotted box (shown with black arrows) shows the same footpoint region as shown in Fig. 5.

spectroscopic study of a recurrent active region jet. The peak DEM, EM, and filling factor values obtained using coronal abundances were found to be a factor of four lower than the values obtained using the photospheric abundances (see Table 5).

The AIA $131 \AA$ image showed a similar structure and morphology of the jet to the EIS Fe VIII $(\lambda 186.605 ; \log T[\mathrm{~K}]=5.8)$ observations (see Fig. 6). We confirm that the emission observed in the spire and the footpoint in the AIA $131 \AA$ images has a main contribution from the lower temperature Fe VIII line. We obtained an initial velocity of $524 \mathrm{~km} \mathrm{~s}^{-1}$ for the jet observed at 14:58 UT from the time-distance analysis (see Sect. 3.5).

A recent multiwavelength study of 20 AR jets by Mulay et al. (2016) showed that most of the AR jets originated at the western periphery of the active region in the vicinity of sunspots and that they were temporally associated with nonthermal type-III radio bursts. The energetic particles gyrating along the open magnetic field structures generally produce nonthermal type-III bursts in the radio dynamic spectrum. Using a potential field source surface (PFSS) technique, authors investigated the spatial co-relation between the AR jets and type-III radio bursts and confirmed the presence of open magnetic field lines at the same region of the jet footpoint. The velocities and DEMs found in the current analysis are consistent with their results.

There are very few simulations of jets, which give actual quantitative values for the plasma properties, that can be compared to observations. Most simulations tend to predict much higher temperatures and lower densities than we observed, however most of them related to coronal hole jets. Two studies addressed active region jets.

Gontikakis et al. (2009) studied the interaction of an emerging bipole and small active region by performing 3D MHD numerical simulations. They reported a hot $(\sim 2 \mathrm{MK})$ and high 
velocity $\left(V=\sim 100 \mathrm{~km} \mathrm{~s}^{-1}\right)$ bidirectional flows as a result of reconnection. They also observed a change in the shape and direction of the jet during the process. They discussed two scenarios of the jet event: firstly, they observed a L-shaped reconnection jet moving with speeds of about $100 \mathrm{~km} \mathrm{~s}^{-1}$ and reached a temperature around $1 \mathrm{MK}$. Later, the jet was found to be trapped in the ambient field and adopted an arc-like shape. The jet started moving laterally and reached a temperature of about $2 \mathrm{MK}$. The authors found good qualitative and quantitative agreement between observations and simulations.

Archontis et al. (2010) studied the long-term evolution of a similar system (a recurrent active region jet) by solving the timedependent, resistive MHD equations in 3D. The authors reported recurrent jets that occurred in direction perpendicular to each other as a result of repeated reconnection events. The recurrent jets appeared to have different physical properties and it changed over the period of time. During the evolution, the authors reported an enhancement of the temperature along the reconnection outflow and they also observed spikes along the leading edge of the jet, which further moved along the parallel field lines. The successive reconnection events were found to be less effective than the previous one and eventually the system attained an equilibrium.

From our spectroscopic and imaging observations, we have seen an inverted-Y topology at the footpoint of the jet and an arcshaped jet spire (see online Movie 1). Our observations show good agreement with the geometric shape of the jet and temperatures $(\sim 2 \mathrm{MK})$ seen in the numerical simulations. However, the bi-directional flow was not observed during the recurrent phase of the jet. The measured densities (spire $=10^{10}$ and footpoint $\left.=10^{11}\right)$ and velocities $\left(\sim 524 \mathrm{~km} \mathrm{~s}^{-1}\right)$ are found to be higher than the values reported in both simulations.

It remains to be seen with new numerical simulations, which will be the subject of a future paper, if such dynamic behaviour can be explained within the standard reconnection scenario associated with flux emergence.

It is important to note that AR jets are relatively common and the fact that there is strong evidence that they occur in regions that are open to the heliosphere (Innes et al. 2011; Chandra et al. 2015; Mulay et al. 2016) makes them one of the best candidates for future detailed studies with the Solar Orbiter and Solar Probe plus suite of remote-sensing and in-situ instruments. Further detailed studies of these events are therefore very useful to prepare for future observations with these missions.

Acknowledgements. The authors are grateful to the referee for comments that helped improve the manuscript significantly. S.M.M. acknowledges support from the Cambridge Trust, University of Cambridge, UK. HEM and G.D.Z. acknowledge the support of STFC. The authors also thank Mr. Paul Wright from
University of Glasgow, UK for his valuable comments. AIA data are courtesy of SDO (NASA) and the AIA consortium. CHIANTI is a collaborative project involving George Mason University, the University of Michigan (USA), and the University of Cambridge (UK). Hinode is a Japanese mission developed and launched by ISAS/JAXA, with NAOJ as domestic partner and NASA and STFC (UK) as international partners. It is operated by these agencies in co-operation with ESA and NSC (Norway).

\section{References}

Archontis, V., Tsinganos, K., \& Gontikakis, C. 2010, A\&A, 512, L2 Aschwanden, M. J., Boerner, P., Schrijver, C. J., \& Malanushenko, A. 2013, Sol. Phys., 283, 5

Asplund, M., Grevesse, N., Sauval, A. J., \& Scott, P. 2009, ARA\&A, 47, 481

Chandra, R., Gupta, G. R., Mulay, S., \& Tripathi, D. 2015, MNRAS, 446, 3741 Chen, N., Ip, W.-H., \& Innes, D. 2013, ApJ, 769, 96

Cheng, X., Zhang, J., Saar, S. H., \& Ding, M. D. 2012, ApJ, 761, 62

Chifor, C., Isobe, H., Mason, H. E., et al. 2008a, A\&A, 491, 279

Chifor, C., Young, P. R., Isobe, H., et al. 2008b, A\&A, 481, L57

Culhane, J. L., Harra, L. K., James, A. M., et al. 2007, Sol. Phys., 243, 19

Del Zanna, G. 2013a, A\&A, 555, A47

Del Zanna, G. 2013b, A\&A, 558, A73

Del Zanna, G., O’Dwyer, B., \& Mason, H. E. 2011, A\&A, 535, A46

Del Zanna, G., Dere, K. P., Young, P. R., Landi, E., \& Mason, H. E. 2015, A\&A, 582, A56

Dere, K. P., Landi, E., Mason, H. E., Monsignori Fossi, B. C., \& Young, P. R. 1997, A\&AS, 125, 149

Feldman, U. 1992, Phys. Scr., 46, 202

Freeland, S. L., \& Handy, B. N. 1998, Sol. Phys., 182, 497

Golub, L., Deluca, E., Austin, G., et al. 2007, Sol. Phys., 243, 63

Gontikakis, C., Archontis, V., \& Tsinganos, K. 2009, A\&A, 506, L45

Innes, D. E., Cameron, R. H., \& Solanki, S. K. 2011, A\&A, 531, L13

Innes, D., Bucik, R., Guo, L.-J., \& Nitta, N. 2016, Astron. Nachr., 337, 1024

Kayshap, P., Srivastava, A. K., \& Murawski, K. 2013, ApJ, 763, 24

Kim, Y.-H., Moon, Y.-J., Park, Y.-D., et al. 2007, PASJ, 59, S763

Kobelski, A. R., Saar, S. H., Weber, M. A., McKenzie, D. E., \& Reeves, K. K. 2014, Sol. Phys., 289, 2781

Kosugi, T., Matsuzaki, K., Sakao, T., et al. 2007, Sol. Phys., 243, 3

Kundu, M. R., Raulin, J. P., Nitta, N., et al. 1995, ApJ, 447, L135

Lee, K.-S., Innes, D. E., Moon, Y.-J., et al. 2013, ApJ, 766, 1

Lemen, J. R., Title, A. M., Akin, D. J., et al. 2012, Sol. Phys., 275, 17

Madjarska, M. S. 2011, A\&A, 526, A19

Matsui, Y., Yokoyama, T., Kitagawa, N., \& Imada, S. 2012, ApJ, 759, 15

Mulay, S. M., Tripathi, D., Del Zanna, G., \& Mason, H. 2016, A\&A, 589, A79

Narukage, N., Sakao, T., Kano, R., et al. 2011, Sol. Phys., 269, 169

Nitta, N. V., Mason, G. M., Wang, L., Cohen, C. M. S., \& Wiedenbeck, M. E. 2015, ApJ, 806, 235

O’Dwyer, B., Del Zanna, G., Mason, H. E., Weber, M. A., \& Tripathi, D. 2010, A\&A, 521, A21

Pottasch, S. R. 1963, ApJ, 137, 945

Weber, M. A., Deluca, E. E., Golub, L., \& Sette, A. L. 2004, in Multi-Wavelength Investigations of Solar Activity, eds. A. V. Stepanov, E. E. Benevolenskaya, \& A. G. Kosovichev, IAU Symp., 223, 321

Yang, L.-H., Jiang, Y.-C., Yang, J.-Y., et al. 2011, RA\&A, 11, 1229

Zhang, Q. M., \& Ji, H. S. 2014a, A\&A, 561, A134

Zhang, Q. M., \& Ji, H. S. 2014b, A\&A, 567, A11 


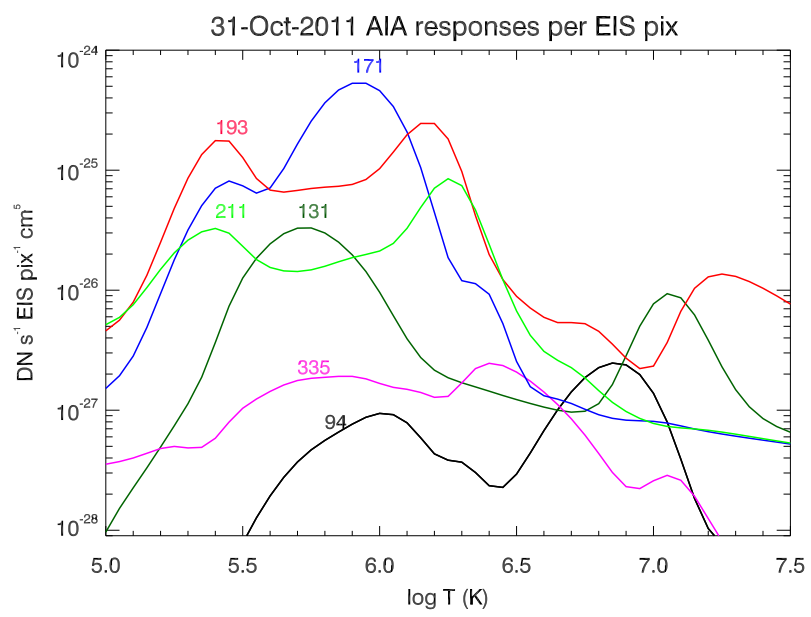

Fig. A.1. AIA temperature responses (per EIS pixel) for the jet spire calculated using the CHIANTI v.8 (Del Zanna et al. 2015) atomic database. We used an electron density of $N_{\mathrm{e}}=7.6 \times 10^{10} \mathrm{~cm}^{-3}$ and photospheric abundances by Asplund et al. (2009).

\section{Appendix A: AIA responses and synthetic spectrum}

The AIA and XRT temperature responses were used in the DEM calculation (refer Sect. 3.6.2).

Figures A.3 and A.4 show synthetic spectra for the SW and LW channel obtained from the EIS DEM and AIA-XRT DEM for the region of the jet spire. Figures A.5 and A.6 show synthetic spectra for the region of the jet footpoint.

\section{A.1. Cross-calibration of AIA and EIS using Moss region}

We selected a moss region to investigate the cross-calibration of the AIA and EIS instruments (shown as yellow box in

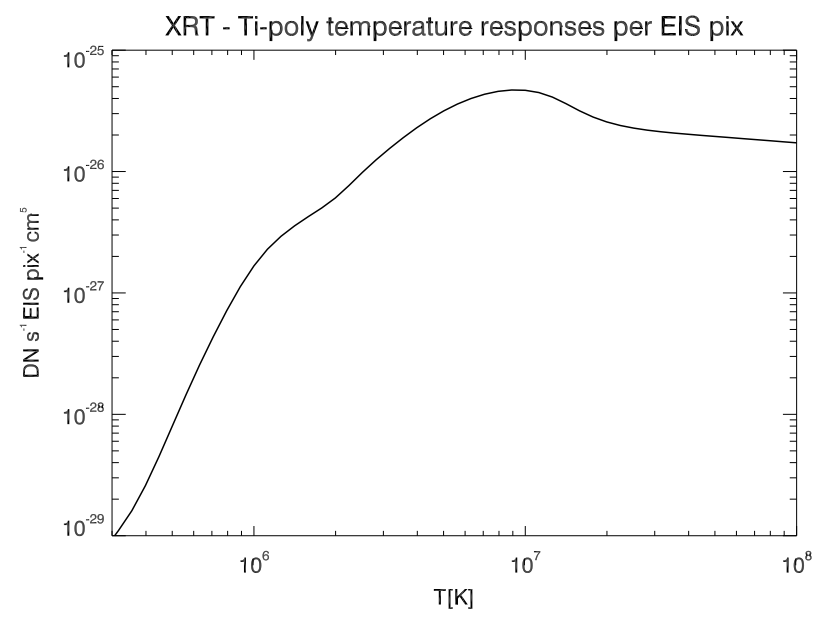

Fig. A.2. XRT temperature responses (per EIS pixel) for the jet spire calculated using the CHIANTI v.8 (Del Zanna et al. 2015) atomic database. We used an electron density of $N_{\mathrm{e}}=7.6 \times 10^{10} \mathrm{~cm}^{-3}$ and photospheric abundances by Asplund et al. (2009).

Fig. 1). This region was used to verify our method of analysis (see Sect. 4). We performed a similar analysis shown in Sect. 3.6 on the moss region. We obtained a light curve (see Fig. A.7) from all AIA channels and found that the moss region was not varying over the period of observations. The AIA-XRT DEM curves (see Fig. A.8) and EIS (see Fig. A.9) in the moss region were consistent over the temperature range from $\log T[\mathrm{~K}]=5.8$ to $\log T[\mathrm{~K}]=6.6$. The total count rates in each AIA channel obtained from the EIS and AIA-XRT DEMs are also found to be in agreement (see Table A.1). So, we have confidence in the calibration of EIS, AIA, and XRT. These results also confirm that the method we used in Sect. 3.6 for the DEM analysis combining AIA and XRT observation is reliable. 
S. M. Mulay et al.: Temperature and density structure of a recurring active region jet
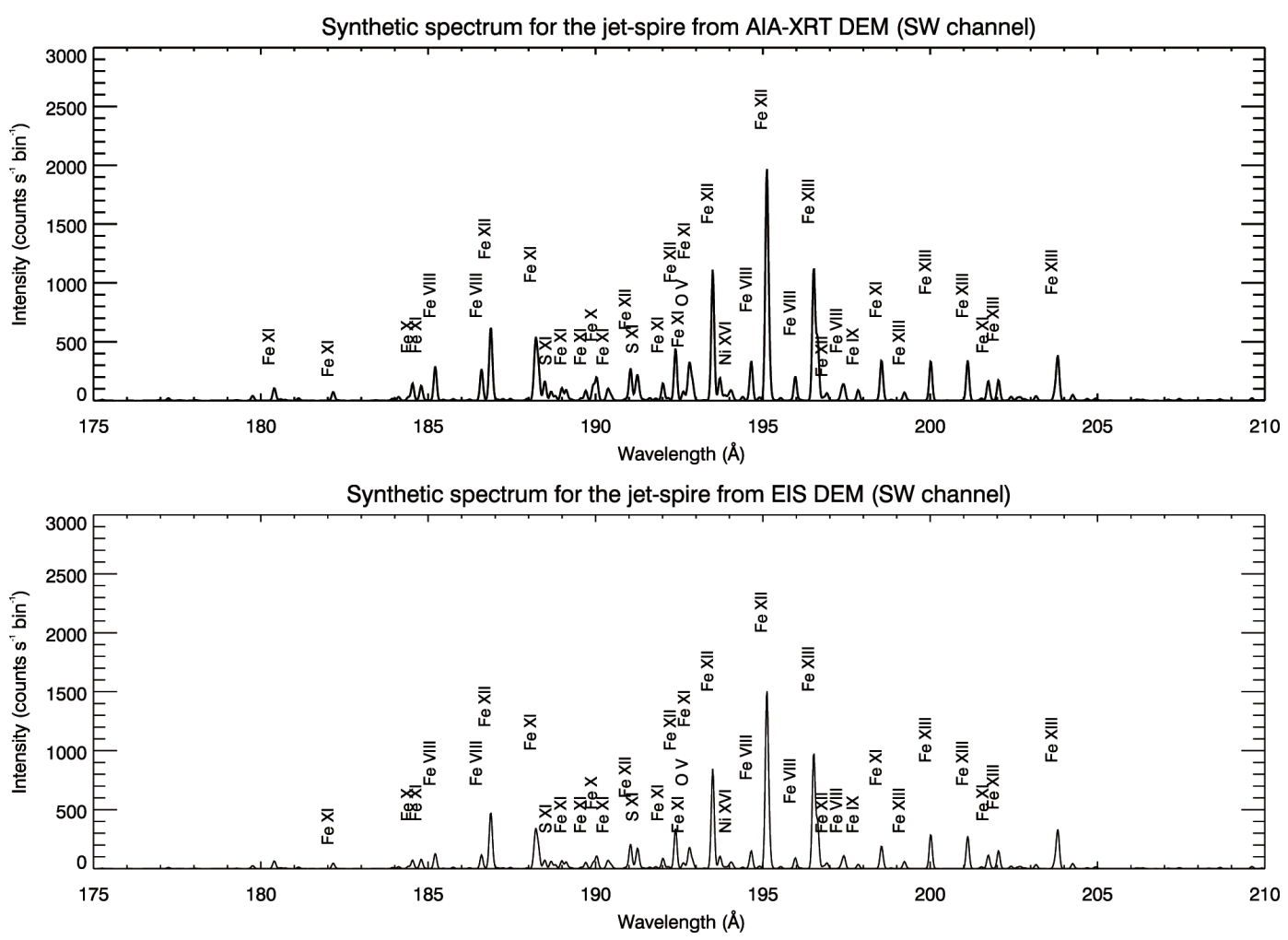

Fig. A.3. Synthetic spectra in short wavelength channel for the jet spire obtained from AIA-XRT DEM curves (top panel) and the EIS DEM curves (bottom panel).
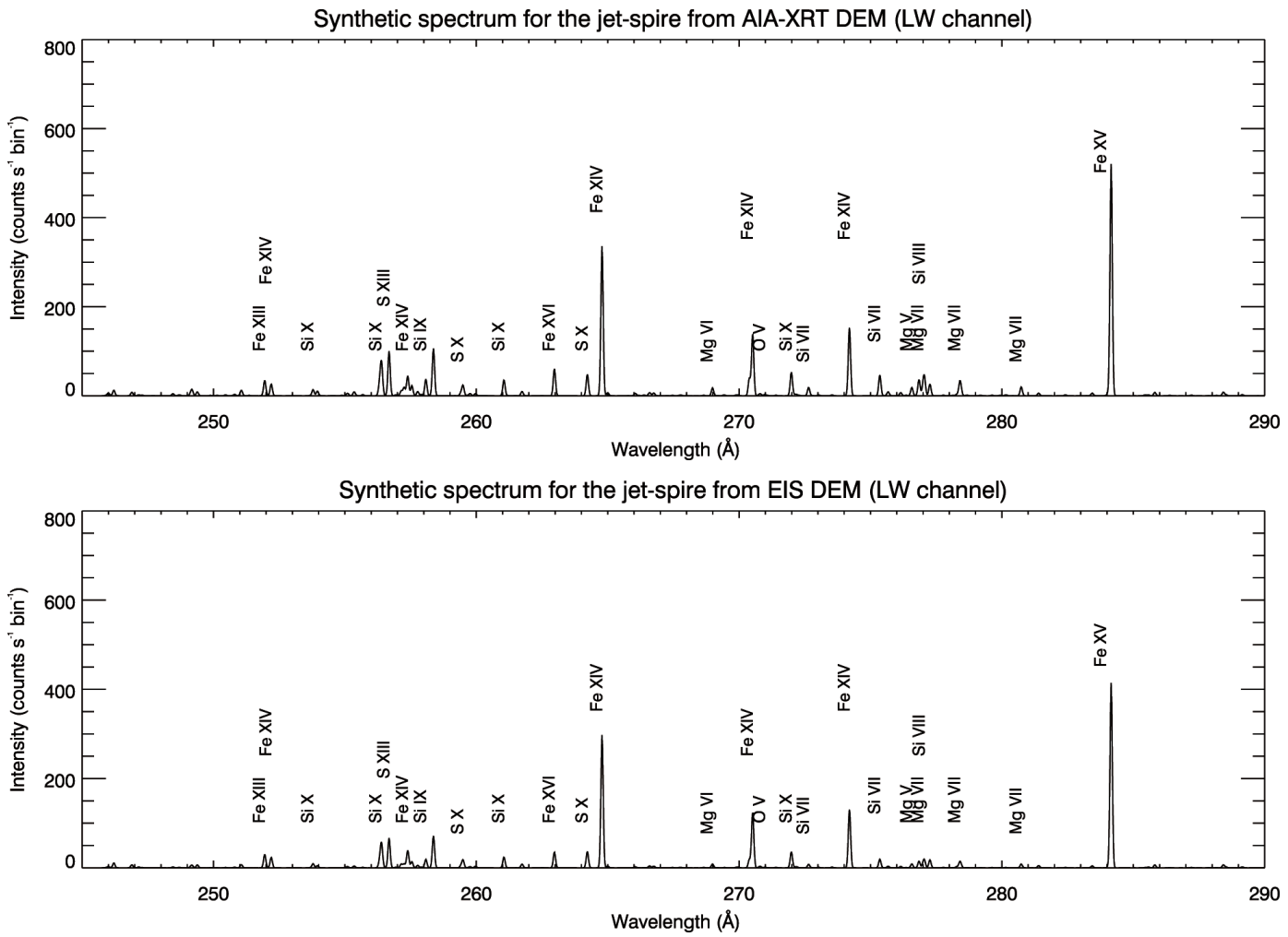

Fig. A.4. Synthetic spectra in long wavelength channel for the jet spire obtained from AIA-XRT DEM curves (top panel) and from the EIS DEM curves (bottom panel). 
A\&A 598, A11 (2017)
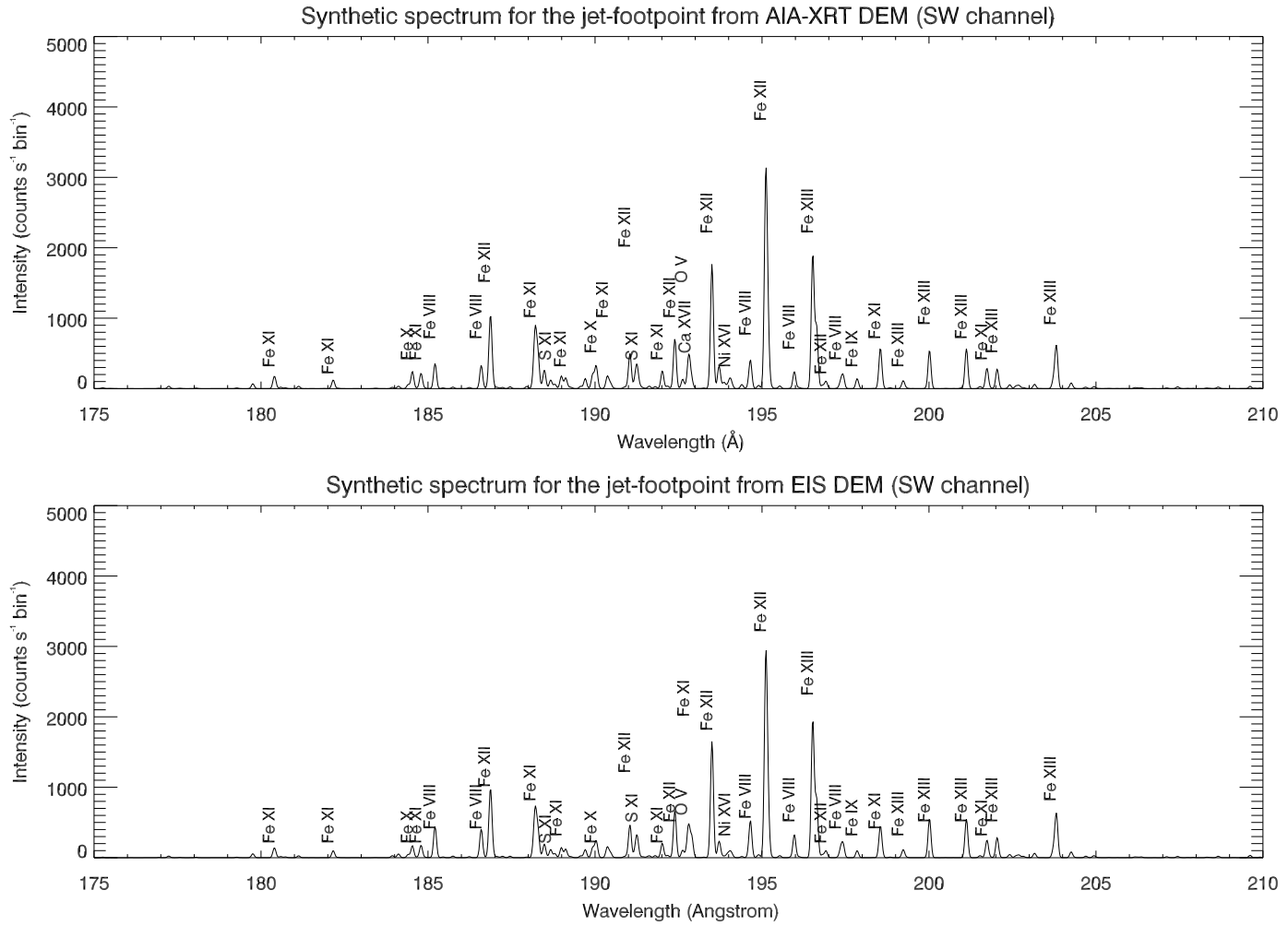

Fig. A.5. Synthetic spectra in short wavelength channel for the jet footpoint obtained from AIA-XRT DEM curves (top panel) and from the EIS DEM curves (bottom panel).
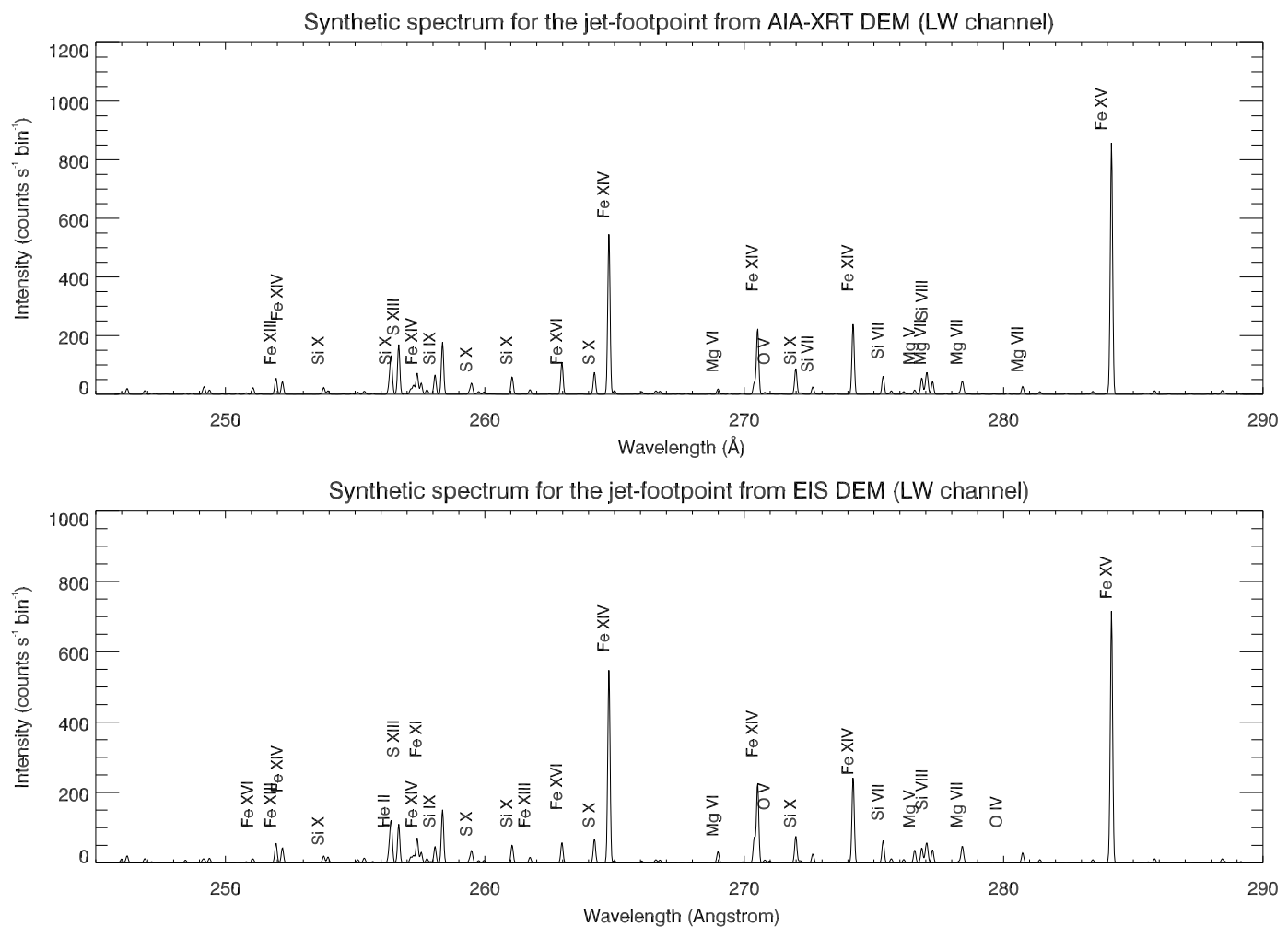

Fig. A.6. Synthetic spectra in long wavelength channel for the jet footpoint obtained from AIA-XRT DEM curves (top panel) and from the EIS DEM curves (bottom panel). 
S. M. Mulay et al.: Temperature and density structure of a recurring active region jet

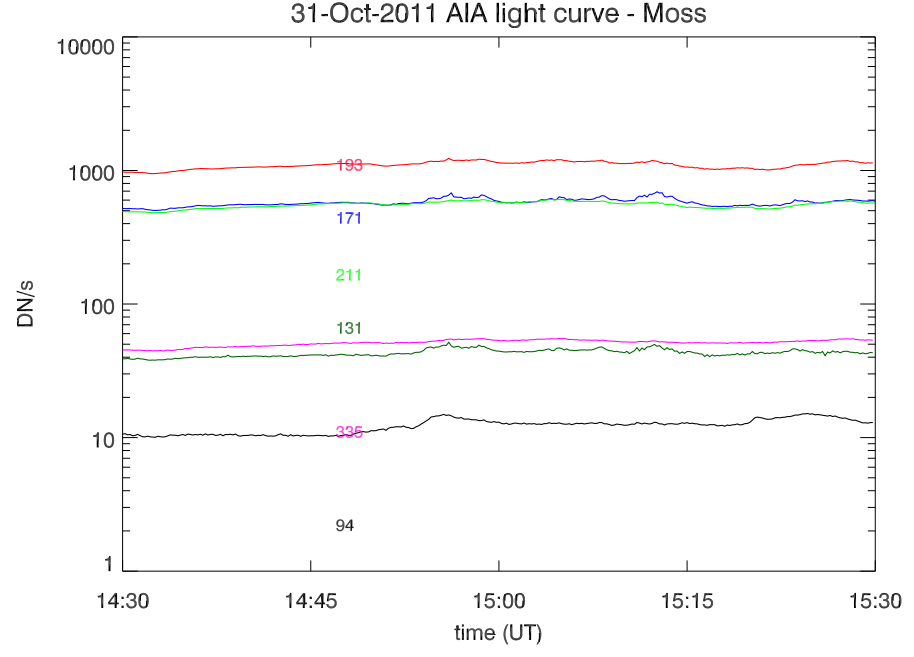

Fig. A.7. Temporal evolution of the moss region calculated in the small yellow box shown in Fig. 1.

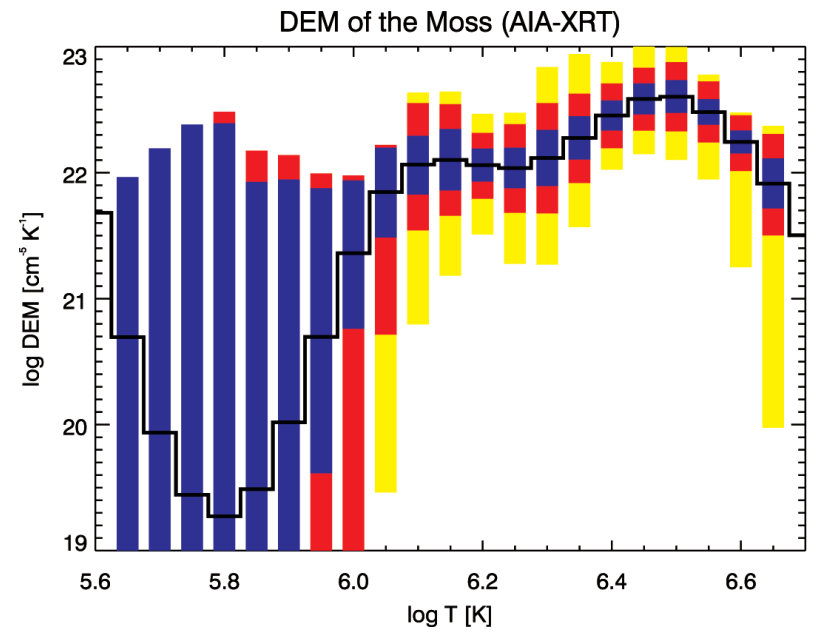

Fig. A.8. AIA-XRT DEM curve for the moss region. The blue rectangles represent $50 \%$, red rectangles $80 \%$, and yellow rectangles $95 \%$ of the MC solutions in each temperature bin.

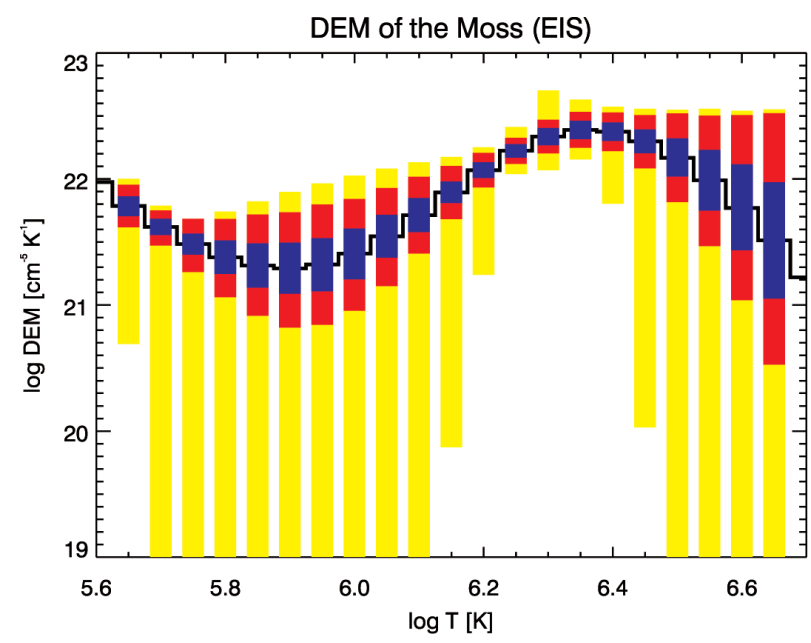

Fig. A.9. EIS DEM curve for the moss region. The blue rectangles represents $50 \%$, red rectangles $80 \%$, and yellow rectangles $95 \%$ of the MC solutions in each temperature bin.
Table A.1. Observed and predicted total count rates in each AIA channel obtained from the DEM in the moss region shown as a yellow box in Fig. 1.

\begin{tabular}{cccc}
\hline \hline $\begin{array}{c}\text { Band } \\
(\AA)\end{array}$ & Observed & $\begin{array}{c}\text { Predicted } \\
(\text { AIA-XRT DEM) }\end{array}$ & $\begin{array}{c}\text { Predicted } \\
(\text { EIS DEM) }\end{array}$ \\
\hline AIA 94 & 34 & $28(-18 \%)$ & $28(-18 \%)$ \\
AIA 131 & 122 & $45(-63 \%)$ & $66(-46 \%)$ \\
AIA 171 & 1629 & $1518(-7 \%)$ & $1522(-7 \%)$ \\
AIA 193 & 3162 & $3020(-5 \%)$ & $2636(-17 \%)$ \\
AIA 211 & 1574 & $1495(-5 \%)$ & $1378(-13 \%)$ \\
AIA 335 & 146 & $137(-6 \%)$ & $102(-30 \%)$ \\
\hline
\end{tabular}

Notes. Column 2 indicates the observed AIA count rates (averaged $\mathrm{DN} / \mathrm{s}$ per EIS pixel). Column 3 shows the predicted total count rates, obtained from the AIA-XRT DEM, and Col. 4 indicates the predicted total count rates from the EIS DEM. Values in parenthesis in Cols. 3 and 4 show the percentage differences between observed and predicted intensities. 